Reconsidering the automaticity of visual statistical learning

Kevin D. Himberger ${ }^{1}$, Amy S. Finn ${ }^{2}$, Christopher J. Honey ${ }^{1}$

${ }^{1}$ Department of Psychological \& Brain Sciences, Johns Hopkins University, Baltimore, MD, USA

5

${ }^{2}$ Department of Psychology, University of Toronto, Toronto, ON, Canada

6

8 The authors gratefully acknowledge the support of the National Institutes of Mental Health 9 (R01MH119099 to CJH); the NVIDIA Corporation (GPU Grant to CJH); the Sloan Foundation 10 (Research Fellowship to CJH); the Canada Foundation for Innovation and Ontario Research 11 Fund (34947 to ASF); the Natural Sciences and Engineering Research Council Discovery Grant 12 (RGPIN-2016-05 to ASF); and the Social Sciences \& Humanities Research Council Insight 13 Program (435171493 to ASF). We thank Chaz Firestone for his insightful feedback and 14 discussion.

15

16

17

18

19 Word Count: 10,294

20 
Abstract

2 Statistical learning refers to the process of extracting regularities from the world without

3 feedback. What are the necessary conditions for statistical learning to arise? It has been argued

4 that visual statistical learning (VSL) is "automatic", such that subjects will passively and even

5 unconsciously extract statistical regularities from streams of visual input as long as they attend

6 to the stimuli. In contrast, our data indicate that simply attending to stimuli is not, on its own,

7 sufficient for learning.

8 In Experiments 1 \& 2, we provided incidental exposure to regularities in a stream of images and

9 observed little to zero VSL across a range of conditions. In Experiment 3, we found that explicitly

10 instructing participants to seek regularities dramatically improved their performance on direct

11 measures of learning, but not on an indirect response time measure. Finally, in Experiments 4 \&

125 , we demonstrated that a methodological confound in prior work using the indirect response

13 time measure could account for some previous evidence of automatic and implicit VSL.

14 Overall, we found very little evidence of learning using direct measures of VSL, and no evidence 15 of learning using an indirect response time measure. Participants who recognized visual 16 sequence regularities in a forced-choice task could also often recreate the sequences when 17 explicitly probed, indicating their knowledge was not entirely implicit. We suggest that some 18 form of active engagement with stimuli may be needed to extract sequential regularities, and 19 that VSL does not occur automatically. 


\section{Reconsidering the automaticity of visual statistical learning}

Humans can rapidly extract regularities from their environment (Saffran, Newport, Aslin, Tunick, \& Barrueco, 1997; Turk-Browne, 2012). For example, we can segment words in a new language by extracting the regularities in the sounds of that language (Saffran, Aslin, \& Newport, 1996; Saffran \& Kirkham, 2018). Similarly, our ability to learn reliable visual patterns over time, such as sequences of landmarks along a route, or features in a stream of photographs, is thought to depend on visual statistical learning. Statistical learning is observed across all ages, from infants to seniors (Kirkham, Slemmer, \& Johnson, 2002; Campbell, Zimerman, Healey, Lee, \& Hasher, 2012), and is thought to occur incidentally (without any conscious intention to learn regularities) (Saffran et al., 1997; Turk-Browne, Scholl, Johnson, \& Chun, 2010). Visual statistical learning has also been proposed as a ubiquitous and inescapable process for reorganizing our brains and minds in response to environmental statistics (P. J. Reber, 2013).

Is visual statistical learning (VSL) an automatic learning process? In standard VSL paradigms, participants are passively exposed to sequential regularities (e.g. images are presented in groups of three, always presented in the same order and interleaved with other groups of three). Because regularities are thought to be "soaked up" by "mere exposure" (Aslin, 2017), participants are often asked simply to attend to the stimuli, or to perform an incidental cover task that is unrelated to the sequence structure. This passive exposure distinguishes standard VSL tasks from more active paradigms, such as artificial grammar learning (A. S. Reber, 1967) and the serial reaction time task (Nissen \& Bullemer, 1987), in which, in order to complete their task, participants continually generate actions or decisions that follow the same regularities that are later tested (as noted in Olson \& Chun, 2001). Attending to the stimuli is necessary for 
1 visual (Turk-Browne, Jungé, \& Scholl, 2005; Toro, Sinnett, \& Soto-Faraco, 2005) and auditory

2 statistical learning (Fernandes, Kolinsky, \& Ventura, 2010) to occur in adults, but it remains

3 unclear whether VSL is automatic when attention is passive (i.e. when participants attend to the

4 stimulus features, but do not have to deeply process them, or make a decision that follows the

5 regularities). In other words, is passive attention sufficient to support VSL? Or is some more active

6 form of processing necessary for learning? For example, participants might need to process the

7 individual stimuli more deeply (Craik \& Tulving, 1975) and / or attend to the relationship between

8 stimuli. We investigated the automaticity of regularity learning in adults by varying the exposure

9 to regularities by either (i) incidentally exposing participants to regularities or (ii) explicitly

10 instructing participants to seek out regularities.

We first measured VSL of sequences of fractal images (Exp. 1) and shape images (Exp. 2)

12 under incidental exposure conditions. In contrast to our pre-registered hypotheses and previous

13 reports, participants exhibited weak evidence of learning using two direct measures (two-

14 alternative forced choice of sequence familiarity and creation of the regularities), and they

15 exhibited no evidence of learning using an indirect measure (response time facilitation). To

16 demonstrate that the nature of engagement with the stimulus stream affects VSL, we provided

17 participants with explicit instruction on the regularities. This explicit regularity seeking produced

18 a very large increase in our direct measures of sequence knowledge, without any change in our

19 indirect measure of learning (Exp. 3). Finally, we discovered that previous reports of learning via

20 this indirect measure (response time facilitation) could be explained by a confound between

21 triplet position and test-stream position. Using our counterbalanced design, there was no 
1

evidence of VSL from response time measures (Exps. 1-3), and when we introduced this confound, the ostensible signature of learning emerged (Exps. 4 \& 5).

Our findings suggest that visual statistical learning may not be as automatic as commonly envisaged (e.g. Turk-Browne et al., 2005; Kim, Seitz, Feenstra, \& Shams, 2009). In our data, we only observe learning under conditions of more active engagement with the stimuli. Under conditions of incidental exposure, we observed weak evidence of VSL via sequence familiarity and creation tests, and we found no evidence of VSL in response time measurements.

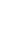

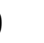

\section{Experiment 1}

Here we investigated the conditions under which visual statistical learning occurs in adults, and the kinds of knowledge it engenders. Because statistical learning appears to generate a combination of implicit and explicit knowledge (Batterink, Reber, Neville, \& Paller, 2015), we compared how learning is expressed given different amounts of exposure $(0,60$, or 96 exposures per triplet) and different measures of learning (e.g. accurate judgments vs. response speeding). Consistent with prior studies (Batterink, Reber, Neville, et al., 2015; Batterink, Reber, \& Paller, 2015; Bays, Turk-Browne, \& Seitz, 2015), we reasoned that if VSL depends on a singular underlying mechanism or process (Kirkham et al., 2002; Perruchet \& Pacton, 2006; c.f. Frost, Armstrong, Siegelman, \& Christiansen, 2015), then learning outcomes should be correlated across distinct measurements within the same participant. However, if statistical learning draws on multiple mechanisms, then a participant might express learning under one measure, but not under another measure. We used one indirect (Olson \& Chun, 2001; Turk-Browne et al., 2005; 
1 Kim et al., 2009; Bertels, Franco, \& Destrebecqz, 2012; Batterink, 2017) and two direct (Saffran,

2 Newport, \& Aslin, 1996; Fiser \& Aslin, 2002; Turk-Browne et al., 2005; Schapiro, Kustner, \& Turk-

3 Browne, 2012; Otsuka, Koch, \& Saiki, 2016) dependent measures to assess the learning of visual

4 regularities within each subject: a target detection (TD) task, a two-alternative forced choice

5 (2AFC) task, and a triplet creation (Creation) task (Fig. 1B). As shown previously in an auditory

6 paradigm (Batterink, Reber, Neville, et al., 2015), dissociable learning processes can be teased

7 apart with the careful selection of post-exposure measures such as these.

8 In sum, we measured VSL under conditions of passive exposure and characterized the

9 relationship between three different measures of learning, to better understand the unitary or

10 multi-component nature of VSL. Our experimental design, hypotheses, exclusion criteria, and

11 analyses were pre-registered using the Open Science Framework (https://osf.io/vtmpb).

Method

Participants. 360 participants were analyzed, with 120 participants in each of three conditions. As documented in our pre-registration, we performed a power analysis using pilot data and estimated our power at greater than 99\% when collecting 120 participants in each condition. Each participant was assigned to either the "0 Exposure", "60 Exposures", or "96 Exposures" condition. Exposure amount was defined by how often each triplet was shown in the

19 “Initial Exposure" phase: 0, 60 or 96 times. Participants were recruited to participate via an 20 advertisement on Amazon's Mechanical Turk platform, with the experiment coordinated via 21 psiTurk 2.1.2 (Gureckis et al., 2016). More detailed recruitment, payment, and demographics are 22 shown in Supp. Table 1 (with age groups and genders shown in Supp. Fig. 1). 
Exclusion Criteria. Online participants require greater compliance and comprehension testing than in-lab participants (Kraut et al., 2004). 602 subjects participated, with 157 subjects excluded and 85 held out to ensure that we had counterbalanced datasets in each condition. The

4 Based upon our pre-registered exclusion criteria (Supp. Table 1), we excluded participants for:

5 incorrectly recorded data $(n=1)$, not performing the cover task with at least $50 \%$ accuracy $(n=23)$, not performing the target detection task with at least $50 \%$ accuracy $(n=10)$, changing windows away from the experiment $>20$ times $(n=22)$, indicating not all stimuli were seen $(n=7)$, indicating previous participation in a similar experiment $(n=13)$, excessive keypresses (e.g. pressed the space bar repeatedly) ( $n=15)$, not making a selection in at least $95 \%$ of 2 AFC test trials $(n=21)$,

10 indicating at least one set of instructions were not understood $(n=27)$, not following instructions

$11(n=15)$, or taking an excessive amount of time to complete the experiment $(n=3)$.

Stimuli. Within each condition, each participant saw a "stimulus set" composed of twelve

13 unique images (Fig. 1A; Schapiro, Kustner, \& Turk-Browne, 2012). The 12 images were organized

14 into 4 sets of three images (i.e. triplets). Thus, we can label the triplets in a stimulus set as A, B, $15 C$ and $D$, with each triplet composed of three "fractal" images: $\left(A_{1}, A_{2}, A_{3}\right) ;\left(B_{1}, B_{2}, B_{3}\right) ;\left(C_{1}, C_{2}\right.$, $\left.16 C_{3}\right) ;\left(D_{1}, D_{2}, D_{3}\right)$. All participants within a condition were exposed to the same 12 images, but the 17 organization of images into triplets was different for each participant within a condition. We counterbalanced the image assignments across subjects within a condition, ensuring that each image appeared equally often (across subjects) as the first, second, or third position of

20 a triplet. This counterbalancing also ensured that triplet position was uncorrelated with image 21 identity across our dataset. 
The same stimulus sets were used across all three exposure conditions $(0,60,96)$. Thus,

for each of the unique assignments of images to triplets and triplet positions that we used, exactly one participant was exposed to that structure in each of the three conditions.

Procedure. Each participant completed the phases of the experiment (Fig. 1B) in the

7 Each phase is described in more detail below.

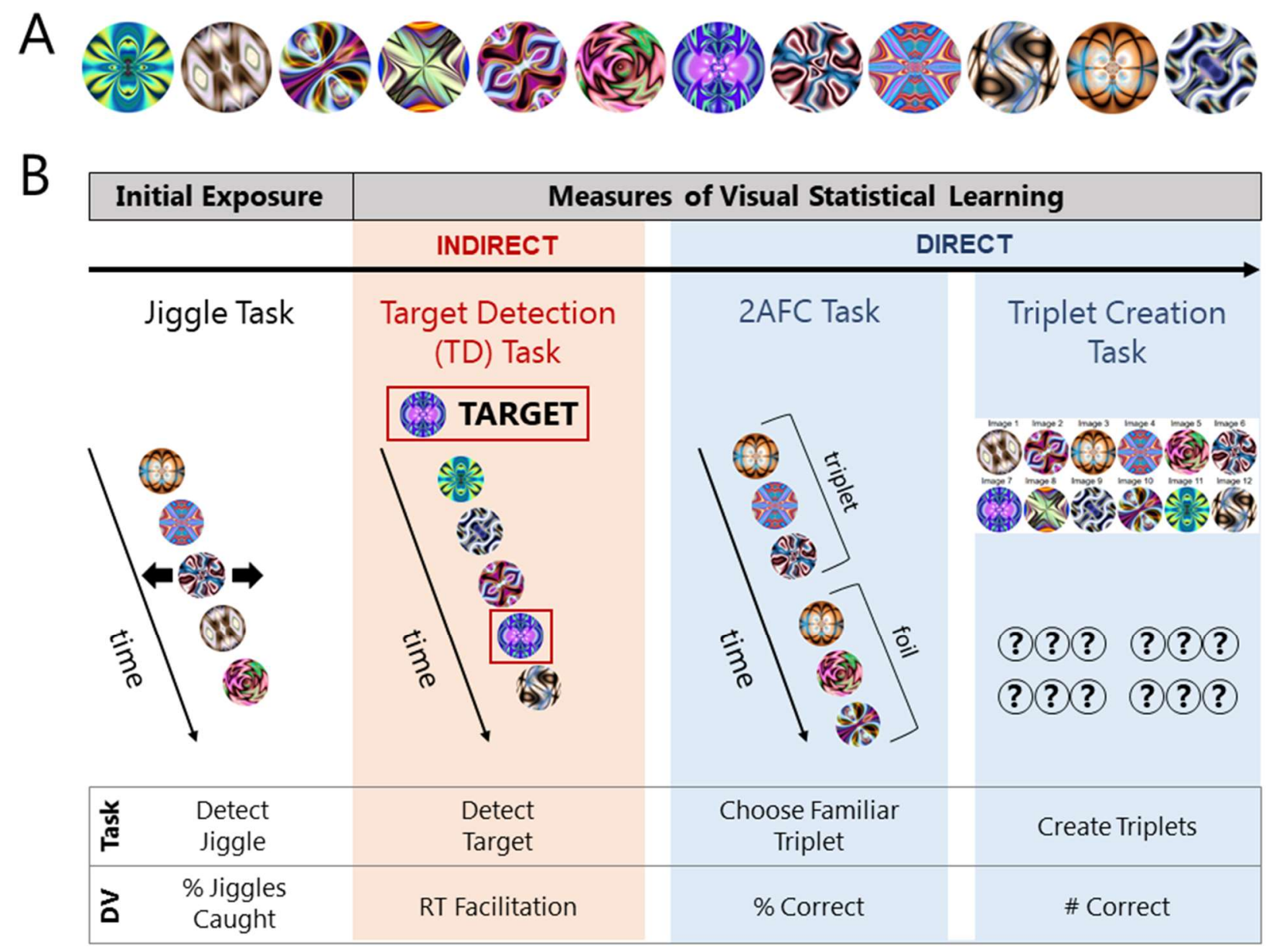

Figure 1. Stimuli \& experimental paradigm. (A) The 12 "fractal" image stimuli used in Experiments 1 \& 3-5. (B) Each column shows a phase of the experiment. The "Task" row indicates the participant's goal in each phase. The "DV" row indicates the primary dependent variable measured in that phase. The experiment was designed so that more direct (more explicit) measures of VSL occurred later. Participants in the "O Exposure" condition skipped the "Initial Exposure" phase. 
Pre-experiment Instructions. After providing informed consent, participants read general

instructions regarding the experiment and its expected duration. Participants were instructed that they should dedicate complete attention to the experiment and to attend to each image.

4 Participants were not told anything about regularities.

Initial Exposure. Participants (except those in the " 0 Exposure" condition) viewed the triplets multiple times (60 or 96) while performing a task in which they were asked to detect

7 jiggles (Turk-Browne, Scholl, Chun, \& Johnson, 2009). Participants in the "0 Exposure" condition

8 proceeded directly from the pre-experiment instructions to the target detection task, skipping 9 the initial exposure.

10 To complete the "Jiggle Task", participants were instructed to detect any transient 11 horizontal motion of an image within a stream of images (i.e. to "catch jiggles"). Participants 12 were instructed to press the space bar whenever a jiggle occurred. The purpose of the Jiggle Task

13 (Fig. 1B, leftmost column) was to diminish the chances that participants would employ explicit

14 strategies to examine the statistical patterns embedded in the stream of images, while ensuring the stimuli were attended. Participants completed practice sessions of the Jiggle Task, initially with feedback, and then without feedback using text instead of the experimental stimuli, until all

17 (four) practice jiggles were detected within 600ms and without feedback. After successful completion of the practice phase, participants performed the Jiggle Task.

During the Jiggle Task, participants viewed a series of polychromatic, abstract images (Fig.

20 1A) presented one-by-one. Images were shown for $800 \mathrm{~ms}$ with an inter-stimulus interval of

$21200 \mathrm{~ms}$. Jiggles began 300ms after stimulus onset with an immediate onset of slight rightward, 22 then leftward, then rightward motion at constant speed (see Supp. Video 1). The duration of 
1 image motion took 200ms. Jiggled images could not occur within 6 images of one another. The exposure phase never began or ended with an image that jiggled. Each image was shown 60 or

396 times (depending upon condition), for a stream of 720 or 1152 images, respectively. The

4 number of jiggles in each condition was equal to the number of exposures per image (i.e. 60 or

5 96), thus each of the twelve images was selected to jiggle 1/12th of the time (e.g. in the "60

6 Exposures" condition, each of the twelve images jiggled 5 times, for 60 jiggles total). The number

7 of items between consecutive jiggles varied pseudo-randomly, ranging from 6 to 31 .

Measures of Visual Statistical Learning. After experiencing either 0,60 or 96 exposures

9 to each triplet during the initial exposure, participants began the test phase. The order of the

10 three measures (Target Detection, 2AFC, and Creation) was fixed to situate the most indirect

11 measure first, with increasingly direct measures following. If participants were exposed to

12 direct measures of learning first, then their performance in the indirect measure could be

13 influenced by explicit knowledge of the structure in the experiment.

Target Detection (TD) Task. The target detection (TD) task (Fig. 1B, second column) tested

participant response speed to targets embedded in a stream of images. The image stream

16 adhered to the same statistical regularities as the initial exposure phase. Each TD trial began by

17 displaying the trial-specific "target", one of the 12 unique images shown during the initial exposure. Participants initiated each trial (i.e. a stream of 7-11 images) by pressing the enter key, then pressing the space bar upon detection of the trial-specific target. The target was shown only

20 once per trial and each of the 12 images served as a target 6 times. Therefore, participants

21 completed 72 TD trials. In keeping with previous work (Turk-Browne et al., 2005; Musz, Weber,

22 \& Thompson-Schill, 2015), presentation was speeded relative to the initial exposure such that 
the stimulus duration was $200 \mathrm{~ms}$ and the inter-stimulus interval (ISI) was $40 \mathrm{~ms}$. Participants were asked to respond quickly and accurately. No practice sessions were performed before the TD task to reduce interference from the introduction of multiple new stimuli.

The positions of targets in each stream were balanced across trials. Following previous work, the target image never appeared in the first or last three positions of a stream (TurkBrowne et al., 2005). Targets could only appear in stream positions 4-8, resulting in stream lengths of 7-11 images. To ensure that any stimulus could appear at any stream position, it was necessary that streams did not always begin with an image that was the first item in a triplet. Thus, the stream position of the target constrained (i) the triplet position of the first item and (ii) the total stream length. For example, if target, $\mathbf{C}_{\mathbf{2}}$, needed to be in stream position 6 , then the stream would need to be 9 elements long (target position plus three) and the stream would need to begin with a triplet position 3 image. Thus, the test stream might be: $A_{3}, B_{1}, B_{2}, B_{3}, C_{1}, C_{2}, C_{3}$, $D_{1}, D_{2}$ (Fig. 5, top). Each image served as the target twice in position 4, and once in all other stream positions $(5,6,7$ or 8$)$. By making targets appear more often in stream position 4 than in other positions, we aimed to mitigate the RT speeding effect that arises from having a nonconstant hazard rate (i.e. having an increasing probability that the target would appear as the next item, given that it had not yet appeared in the stream).

This procedure ensured that all target images (regardless of whether they were in the first, second or third position of their triplet) appeared equally often within a given stream position. In this way, there was no relationship between triplet and stream position.

Two-Alternative Forced Choice Task (2AFC). Before beginning the 2AFC phase, participants were asked if they "noticed any patterns in the stream of images". If they responded 
positively, they were asked to "briefly describe" the patterns. Following this question, all participants were informed that the images they had seen had followed a pattern, and that they would use "familiarity" to choose which of two sequences of images seemed more familiar.

Participants then practiced the 2AFC task. They were told to use the left and right arrow keys to indicate which of two sequences of three images, on the left or right, was "more familiar". On each practice trial, text (instead of images) appeared on either the left or right, indicating which side was the correct choice (e.g. "Choose", "the", "left"). Participants could not proceed past this practice phase until they successfully completed two consecutive practice trials.

The $2 \mathrm{AFC}$ task (Fig. 1B, third column) contained 32 trials. On each trial, participants decided which of two groups of images felt more familiar. Participants initiated each trial by pressing the enter key. On each trial, participants were shown a "real" triplet (i.e. three images shown in the same order they appeared during the initial exposure and the TD task) and a "foil" triplet (i.e. three images never presented in the sequence shown). Images were displayed one at a time (using the same timing as the initial exposure; $800 \mathrm{~ms}$ stimulus duration with a 200ms ISI) with a $1000 \mathrm{~ms}$ inter-triplet delay. The first triplet was displayed on the left side of the screen and the second triplet was displayed on the right side of the screen. Triplet type ("real" or "foil") was counterbalanced such that each type occurred first and second an equal number of times, as described below.

Foil triplets were created by reshuffling "real" triplets while keeping the within-triplet position constant (e.g. triplet: $C_{1}, C_{2}, C_{3}$; foil: $A_{1}, B_{2}, C_{3}$ ). Four foils were created in this way. During the test, the set of four real triplets and four foil triplets was fully crossed, for a total of 16 test pairs. An additional 16 test pairs were created by copying the original 16 test pairs and swapping 


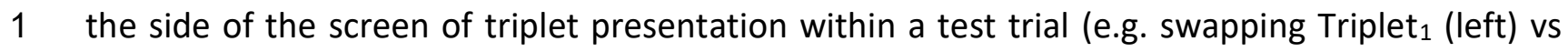

$2 \operatorname{Triplet}_{2}$ (right) to $\operatorname{Triplet}_{2}$ (left) vs $\operatorname{Triplet}_{1}$ (right)), thus eliminating any left-right bias during test.

3 The 32 trials were presented in a pseudo-random order.

4 Creation Task. As the final, and most direct, measure of statistical learning, we asked 5 participants to perform a Creation task (Fig. 1B, fourth column). Participants were informed that 6 the 12 images seen during the initial exposure (60 \& 96 exposure conditions) and during the TD 7 task had been composed of 4 triplets, with the images within each triplet always following one 8 another in the same order. They were then asked to "create" the triplets, by selecting images in 9 order from a spatial array.

10 All 12 images were shown in two rows at the top of the screen, and in a random order for 11 each participant (see Fig. 1B, "Triplet Creation Task"). A number from 1-12 was assigned to each 12 image. Below the array of images were 12 drop-down boxes, organized into sets of three. The 13 options in each drop-down box were images 1-12 (as assigned in the image section). Participants 14 were asked to create four triplets by selecting using the drop down boxes. Each image could only 15 be used once across all drop-down boxes, to ensure that all 12 images were assigned to a unique 16 position. All 12 drop-down boxes required an entry before the participant could submit their 17 selections and proceed to the post-experiment questionnaire. Post-Experiment Questionnaire. Participants were asked to assess their own performance of each of phase of the experiment. They were asked if they knew there were four

20 triplets prior to being told about the triplets in the instructions for the Creation task. They were 21 asked whether they used any strategies in each of the phases. Additionally, they were asked if 
1 they had mentally assigned labels to any of the images and, if so, to list any labels they had 2 assigned.

Data Analyses. The majority of statistical analyses performed were non-parametric. This

4 decision was based upon violations of normality in the data (e.g. Supp. Fig. 9A). This applied to

5 all three dependent variables from our measures of visual statistical learning (Fig. 1B). Therefore,

6 comparisons between triplet positions and the three levels of exposure employed the Kruskal-

7 Wallis $\mathrm{H}$ test as implemented in SciPy 1.2.1 (Jones, Oliphant, Peterson, \& others, 2001).

8 Comparisons across experiments used Mann-Whitney $U$ tests. 95\% confidence intervals were

9 estimated using a bootstrap procedure which, in short, generated multiple mock experiments by

10 sampling the observed data with replacement, then used the critical values at $2.5 \% \& 97.5 \%$ of

11 the resulting, sorted iterations as the interval (see "Bootstrap Procedure" in Supp.).

12 Indirect Measure (Target Detection) Analysis. Learning was assessed by calculating the

13 within-subject difference between response times to all targets in triplet first positions and all

14 targets in triplet third positions. Thus, the response time facilitation (RTF) was quantified as the

15 difference in RT between the first and third triplet positions (RTF 1-3). Permutation tests were

16 used to calculate p-values (see "Permutation Testing" in Supp.).

17 Direct Measures (2AFC \& Creation) Analysis. Performance on the 2AFC task was assessed

18 by calculating the mean percentage of correct choices made when assessing triplet vs. foil groups

19 of images. Performance in the Creation task was assessed by calculating the mean number of

20 triplets created.

21 The distributions of $2 \mathrm{AFC}$ and Creation performance under the null hypothesis were 22 estimated using Monte Carlo simulations (see "Monte Carlo Simulation" in Supp.). Since our pre- 
1

registered hypotheses anticipated learning, we employed one-way tests to determine critical thresholds in the null distribution. P-values were computed by comparing observed means with critical thresholds in the null distribution. If the overall experimental mean exceeded any of the thresholds ( $p<0.05,0.01$ or 0.001$)$ we reported the most unlikely threshold exceeded.

Effect Size Analyses. For Kruskal-Wallis $\mathrm{H}$ tests, we converted the $\mathrm{H}$ statistic (as a proxy for chi-squared) into an approximation of eta squared. Since the number of groups we compare (with number of triplet positions and number of exposure conditions) is always three, the eta squared approximation simplifies to Eq. 1 (Murphy, Myors, \& Wolach, 2014).

$$
(E q .1) \eta^{2}=H /\left(H+n_{\text {within_group }}-n_{\text {groups }}\right)
$$

Pingouin 2.7 (Vallet, 2018) to calculate the rank-biserial correlation (RBC) or the matched pairs rank-biserial correlation (MPRBC; Kerby, 2014), respectively.

\section{Results}

Jiggle Task. Participants performed well in the cover task during their initial exposure to the visual sequences. Stimulus movements ("jiggles") were detected with $90 \%$ accuracy in both the 60 and 96 exposures conditions, and the mean time to detect jiggles was less than 460ms in both conditions (Supp. Table 1). Thus, participants were attending to the stimuli during initial exposure.

Target Detection Task. We observed minimal evidence of learning using the indirect measure (TD task, Fig. 2A). The mean RTF1-3 effects were: $0.23 \mathrm{~ms}(95 \% \mathrm{Cl}:[-0.41,0.96]), 3.2 \mathrm{~ms}$ 
1 (95\% Cl: $[2.55,3.93])$ and $-1.1 \mathrm{~ms}(95 \% \mathrm{Cl}:[-1.8,0.30])$ for the 0,60 , and 96 exposure conditions respectively. If no learning occurred, we would expect no difference between response times to triplet first or third positions, therefore RTF1-3 would be approximately 0ms. This was the case

4 in the 0 Exposure and 96 Exposures conditions. The very small ( $3.2 \mathrm{~ms})$ effect in the 60 Exposures condition was statistically significant $(p<0.001)$ (Table 1, "Mean RTF1-3"). At the same time, a Kruskal-Wallis test revealed no significant differences between response times by triplet position in any condition $\left(\mathrm{H}(2)<1.4\right.$ and $\eta^{2}<0.012$ for all conditions; Table 1, "RT differences between triplet positions"). Since all effect sizes were smaller than $4 \mathrm{~ms}$, and since the condition with most exposures (i.e. 96) provided no evidence of learning, we concluded that there was no meaningful learning-related speeding of response times. Overall, items that began a triplet were detected at the same speed as items that ended a triplet, regardless of the amount of exposure provided to the statistical regularities.

2AFC Task. Forced choice accuracy was low, but exceeded statistical thresholds in two of exposure" condition (mean=51.4\%, 95\% $\mathrm{Cl}=[51.2,51.6], \mathrm{p}=0.047$ ) and in the "96 exposures" condition (mean=51.7\%, 95\% $\mathrm{Cl}=[51.5,51.9], \mathrm{p}=0.021$ ), but not in the "60 exposures" condition

17 (mean=50.2\%, 95\% Cl=[50.0, 50.4]).

Creation Task. Participants created triplets at rates above chance (critical threshold of mean triplets created=0.033) in all conditions ( 0 Exposures: mean=0.15, $\mathrm{p}<0.001, \mathrm{Cl}=[0.14,0.16]$;

2060 Exposures: mean $=0.13, p<0.001, \mathrm{Cl}=[0.13,0.14] ; 96$ Exposures: mean=0.15, $\mathrm{p}<0.001, \mathrm{Cl}=[0.14$,

210.16 ; Fig. 2C; Table 1, "Mean triplets created"), but they also generated the "foil" triplets (from 
suggests that some participants were learning the structure of the foil triplets during the 2AFC test. However, triplets were created more than foils in each condition and a Wilcoxon signedrank test of data combined from all conditions showed that triplets were created at a rate significantly higher than foils ( $W=572, p=0.030, M P R B C=-0.062)$.

Correlations between measures. Consistent with our original hypothesis, the direct learning measurements (from the 2AFC \& Creation tasks) were positively correlated with one another in conditions where participants had initial exposure to the triplet regularities (Spearman rho $=0.15,0.19,0.20$, for the 0, 60 and 96 exposures; Fig. 2D; Table 1). The only non-significant correlation was in the "0 Exposure" condition, where there was no initial exposure. Also as hypothesized, the direct learning measurements were uncorrelated with the indirect learning measurement, RTF1-3, in all conditions (Table 1; Supp. Fig. 3).

Consequences of varying exposure. Across all measures of learning we observed no significant difference in performance between the exposure conditions. We had predicted that increasing the amount of initial exposure to regularities would benefit performance in the direct tasks (Figs. 2B \& 2C) while having little effect on the indirect measure (Fig. 2A). However, we did not observe monotonically increasing performance in the direct tasks as a function of the amount of initial exposure to regularities. We observed no difference between conditions on either direct measure, using a Kruskal-Wallis $H$ test (2AFC: $H(2)=1.5, \eta^{2}=0.013$; Creation: $H(2)=0.039$, $\eta^{2}=0.00033$ ). In addition, there was no change in response time facilitation (RTF1-3) by exposure amount, nor any significant difference between conditions $\left(H(2)=0.88, \eta^{2}=0.0075\right)$.

\section{Discussion}


Conflicting with our pre-registered hypotheses, we found minimal evidence for statistical learning using the indirect target detection (TD) measure and we observed very weak

3 evidence for learning in the 2AFC and Creation tasks. Unlike prior work (Fiser \& Aslin, 2002;

4 Turk-Browne et al., 2005), we found no evidence that increased exposure to statistical

5 regularities significantly improved performance on any measure of statistical learning (see also

6 Finn \& Hudson Kam, 2008). Notably, participants with zero exposure to the triplets prior to test

7 demonstrated equivalent performance to participants who had more than 10 minutes of

8 passive exposure in the Jiggle task (Fig. 2).

As expected, we observed a correlation between the direct measures (2AFC and

10 Creation). The lack of correlation between the direct measures and the indirect measure (TD)

11 was consistent with our hypothesis that statistical learning reflects a mixture of distinct

12 processes. However, because the learning was weak overall, it remains unclear how strongly this

13 evidence could be taken to support the theory of distinct learning processes.

Our observation of overall weak learning, with no effect of the amount of exposure, could

15 indicate that performance was hampered by our constellation of experimental parameters (e.g.

16 stimulus type, method of initial exposure, specific instructions). We therefore investigated our

17 choice of stimulus type (Exp. 2) and the method of initial exposure (Exp. 3) in two follow-up

18 experiments, intending to improve the learning performance across all measures. In Experiment

192 , we used a simpler set of stimuli which produced robust statistical learning in prior studies (Fiser

20 \& Aslin, 2002; Schapiro, Gregory, Landau, McCloskey, \& Turk-Browne, 2014; Schlichting, Guarino,

21 Schapiro, Turk-Browne, \& Preston, 2017). 


\section{Experiment 2}

Might the poorer-than-expected learning in Experiment 1 have resulted from our choice

4 of stimulus? To test this, we designed an experiment identical to the "60 Exposures" condition of

5 Experiment 1, except that we replaced the complex and colorful fractal images with simpler,

6 monochromatic "shape" stimuli. Simple shapes have been used in many prior experiments to

7 demonstrate visual statistical learning (e.g. Fiser \& Aslin, 2001, 2002, 2005; Fiser, Scholl, \& Aslin,

8 2007; Orbán, Fiser, Aslin, \& Lengyel, 2008; Turk-Browne, Isola, Scholl, \& Treat, 2008; Kim et al.,

9 2009; Glicksohn \& Cohen, 2011; Barakat, Seitz, \& Shams, 2013; Schapiro et al., 2014; Bertels et

10 al., 2012; Bertels, Destrebecqz, \& Franco, 2015; Schlichting et al., 2017; Siegelman, Bogaerts, \& 11 Frost, 2017; Siegelman, Bogaerts, Elazar, Arciuli, \& Frost, 2018). Since there was no effect of

12 exposure in Experiment 1, we chose 60 exposures for Experiments 2-5 to remain consistent 13 across our experiments and with the extant literature.

Method

Participants. 120 participants (Supp. Table 1; Supp. Fig. 1) were analyzed from data

17 collected via an advertisement on Amazon's Mechanical Turk platform created using psiTurk

2.2.3. Data from more than 120 participants were collected to ensure counterbalancing between

19 “mini-groups" (as in Exp. 1), with 18 excluded (see "Exclusion Criteria” below) and 38 participants

20 left unanalyzed. Participants received monetary compensation for completing the experiment 21 (Supp. Table 1). 
Exclusion Criteria. 176 subjects participated, with 18 participants excluded for violating one of the exclusion criteria (as in Exp. 1; Supp. Table 1) and 38 participants were left unanalyzed

3 to ensure counterbalancing.

Stimuli. The images were organized into triplets in an identical manner to Experiment 1, except that that the 12 complex fractal stimuli (Fig. 1A) were replaced with 12 simpler black shape stimuli (Supp. Fig. 5, Schapiro et al., 2014).

Procedure. Identical to the "60 exposures" condition of Experiment 1.

\section{Results} indicating that they were paying attention to the stimuli. Despite this excellent performance on the cover task, participants did not significantly improve their learning performance when using shapes rather than fractal images. Compared to the 60-exposures condition in Experiment 1, we observed no significant change in performance on any measure (TD RTF1-3: Mann-Whitney $\mathrm{U}=7676$, Rank Biserial Correlation RBC=-0.066; 2AFC: $\mathrm{U}=6637, \mathrm{RBC}=0.078$; Creation: $\mathrm{U}=7066$, $\mathrm{RBC}=0.019$ ). In the indirect TD task (Fig. 2A), there was no difference in RTs between triplet positions $\left(H(2)=0.11, \eta^{2}=0.00094\right)$. RTs did not decrease by triplet position, resulting in a small negative RT facilitation measurement (RTF1-3 mean=-1.58ms, 95\% Cl=[-2.06, -1.12]; Table 1).

19 Participants performed slightly above chance in the 2AFC (mean=52.2\%, 95\% $\mathrm{Cl}=[52.0,52.4]$, $20 \mathrm{p}<0.01$, Fig. 2B). Similarly, triplet creation was above chance (mean $=0.17,95 \% \mathrm{Cl}=[0.16,0.17]$,

$21 \mathrm{p}<0.001$, Fig. 2C). As in Experiment 1, foil triplets were also created at a rate higher than chance 22 (mean=0.092, 95\% Cl=[0.087, 0.097], $\mathrm{p}<0.001)$, and quantitatively lower than triplet creation, 
1 with no statistical difference between triplets and foils created ( $W=73.5, M P R B C=-0.059$; Supp. Fig. 2; Table 1).

As in the 60 \& 96 exposures conditions from Experiment 1, there was a significant

4 correlation between performance on the direct tasks (2AFC \& Creation: Spearman rho=0.269, $5 \mathrm{p}<0.01$, Fig. 2D). As in all other experiments, no significant relationships existed between indirect and direct tasks (RTF1-3 \& 2AFC: Pearson r=0.034; Supp. Fig. 3A; RTF1-3 \& Creation: Spearman

7 rho=-0.014; Supp. Fig. 3B).
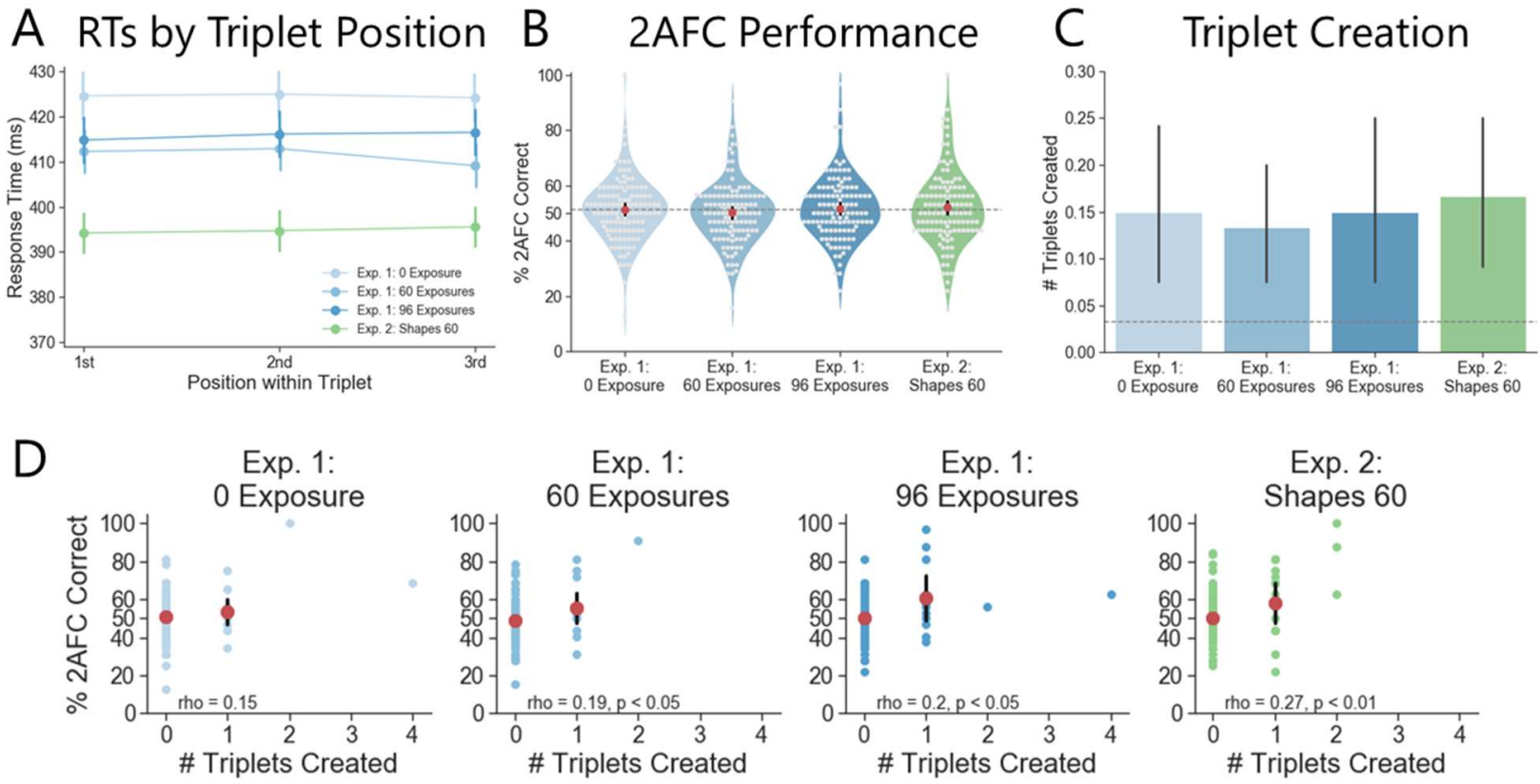

Figure 2. Performance across three measures of statistical learning and correlations between direct measures. (A)

RTs by triplet position. (B \& C) Dashed gray lines indicate chance performance (alpha $=0.05)$. (B) Distribution of

2AFC accuracy across participants. Gray dots indicate a single participant. (C) Mean number of triplets created 
1

\section{Discussion}

Even with a simpler stimulus set, participants demonstrated very little, if any, learning (Fig. 2A-C). While performance on the direct measures was quantitatively higher than Experiment 1, it was not significantly so. Response times in the indirect task were equivalent across all triplet positions, providing no evidence of learning (Fig. 2A). We observed the same pattern of relationships between direct measures and between direct and indirect measures as Experiments $1 \& 2$ (Fig. 2D). In sum, this data suggests that stimulus set did not drive the pattern of the data reported in Experiment 1. Collectively, the data from Experiments 1 \& 2 suggest that common assumptions regarding the ease of VSL during exposure should be revisited.

\section{Experiment 3}

(1)

(1)

The observation of weak (or zero) learning across all three measures might be explained by some property of our initial exposure phase. Therefore, we designed a third experiment which replaced the cover task by telling participants explicitly about the existence of the triplet regularities and providing them with feedback. We reasoned that explicit knowledge should improve performance (at least on the direct measures) and establish a positive control for our measures. Thus, the Jiggle Task was replaced with explicit instructions about the triplet structure, followed by training for participants until they were able to create all four triplets independently. This procedure not only allows us to validate our measures, but also allowed us to determine which of our dependent measures (TD, 2AFC, and Creation tasks) reflect learning when participants have explicit knowledge of the regularities. 


\section{Method}

Participants. 54 participants (Supp. Table 1; Supp. Fig. 1) were analyzed from data

4 collected via an advertisement on Amazon's Mechanical Turk platform created using psiTurk

$5 \quad 2.2 .4$ (Gureckis et al., 2016). We expected to require a smaller sample of participants to reliably

6 detect learning in this condition, given the use of explicit instruction. Additionally, our

7 counterbalancing approach required the number of unique conditions to be a multiple of 3 . We

8 therefore ran multiple participants simultaneously, with data collected until we had three

9 participants in each of 18 unique counterbalanced versions (resulting in data from more than 54

10 participants). Participants received monetary compensation for completing the experiment

11 (Supp. Table 1).

12 Exclusion Criteria. 107 subjects participated, with 45 participants excluded for violating

13 one of the exclusion criteria (Supp. Table 1) and 8 participants were left unanalyzed to ensure 14 counterbalancing.

15 Stimuli. Identical to Experiment 1 (i.e. fractals).

16 Procedure. The procedure was identical to Experiment 1: 60 Exposures (see "Methods" 17 in Exp. 1), except the "Pre-experiment Instructions" and "Initial Exposure" were modified as 18 described below.

Prior to initial exposure, participants were told that: (1) they needed to "quickly and

20 accurately learn four sets of images", (2) images would be "grouped into sets of three (i.e.

21 triplets)", and (3) they needed to pay attention to each image and its position within the triplet. 
1 Their goal was to "learn which images occur together (and in which order)". Participants were

2 advised that naming the images might help them learn the triplets.

3 After reading the instructions, participants began a training phase to learn the four

4 triplets. This phase replaced the Initial Exposure/Jiggle Task from Exp. 1. Participants were shown each image sequentially, one at a time, for $800 \mathrm{~ms}$ with an inter-stimulus interval of $200 \mathrm{~ms}$. The twelve unique images were shown triplet-by-triplet (e.g.

$\left.7 A_{1}, A_{2}, A_{3}, B_{1}, B_{2}, B_{3}, C_{1}, C_{2}, C_{3}, D_{1}, D_{2}, D_{3}\right)$. After seeing each of the 12 images, participants read

8 that they would see the triplets again and were prompted to press a key to initiate the same 9 stream of 12 images.

10 After seeing each of the triplets twice, participants attempted to re-create the four 11 triplets using the same procedure as in the Creation task. Upon submitting their four triplet 12 creations, the participants were shown which triplets were correct and which triplets were 13 incorrect using text above each triplet (on the same screen, in the same spatial layout). If all 14 triplets were created successfully, participants moved on to testing, beginning with the target 15 detection task (as in Exps. 1 \& 2). If any triplets were incorrect, participants were shown the 16 instructions again, were exposed to the images again, and attempted to create the triplets again.

17 Participants were limited to 10 attempts. Instructions for the 2AFC task remained consistent, with participants asked to make judgements based upon familiarity.

\section{Results}


Participants learned the triplet structure quickly and accurately: they successfully created all four triplets after a median of 3 attempts, with no participant requiring more than 7 attempts to achieve perfect creation accuracy.

Following explicit instruction, we observed robust evidence of learning in both of the direct measures (2AFC: mean=81.3\%, 95\% $\mathrm{Cl}=[80.6,82.0]$; Creation: mean=3.30, 95\% $\mathrm{Cl}=[3.25$, 3.34]; Figs. 3B \& 3C). Performance was significantly better relative to matched exposure in Experiments 1 \& 2 (60 Exposures: (2AFC) $U=707, p<0.001, R B C=0.78$, (Creation) $U=828, p<0.001$, $R B C=0.74$; Shapes: $(2 A F C) U=178, p<0.001, R B C=0.95$, (Creation) $U=196, p<0.001, R B C=0.94)$.

Despite the strong evidence of learning in the direct measures, there was no evidence of learning in the indirect target detection task (Fig. 3A; Table 1). As in Experiments $1 \&$ 2, there was no difference in RTs between triplet positions $\left(H(2)=4.28, \eta^{2}=0.00076\right)$. In a direct test comparing the first and third triplet positions, RTs were in fact significantly faster in the first triplet position, indicating a significantly negative RT facilitation measurement (RTF1-3 mean=-10.7ms, 95\% Cl=[-

\section{3, -8.98]; Table 1).}

Unlike Experiments 1 \& 2, foil triplets from the 2AFC task were not created at a rate higher than chance (mean=0.019, 95\% $\mathrm{Cl}=[0.014,0.023])$, and there was a large and significant difference between the number of triplets and foils created $(W=0.0, p<0.001, M P R B C=-0.94$; Supp. Fig. 2; Table 1). These results suggest that when participants have explicit knowledge of the triplet structure prior to the 2AFC task, then the foil triplets seen during 2AFC testing do not generate interference with the true triplets.

The correlation between performance on the direct tasks was nearly zero and not statistically significant, (2AFC \& Creation: Spearman rho=0.016, Fig. 3D), but this likely reflects a 
1 ceiling effect on both of these direct measures of learning. As in all other experiments, there was

2 no significant relationship between indirect and direct measures of learning (RTF1-3 \& 2AFC:

3 Pearson r=0.042; Supp. Fig. 3A; RTF1-3 \& Creation: Spearman rho=0.068; Supp. Fig. 3B).

4

5 Table 1

6 Results from Experiments 1-3

\begin{tabular}{|c|c|c|c|c|c|}
\hline & $\begin{array}{l}\text { Exp. } 1 \text { (0 Fractal } \\
\text { Exposures) }\end{array}$ & $\begin{array}{l}\text { Exp. } 1 \text { (60 Fractal } \\
\text { Exposures) }\end{array}$ & $\begin{array}{l}\text { Exp. } 1 \text { (96 Fractal } \\
\text { Exposures) }\end{array}$ & $\begin{array}{l}\text { Exp. } 2 \\
\text { (Shapes) }\end{array}$ & $\begin{array}{l}\text { Exp. } 3 \text { (Explicit } \\
\text { Instruction) }\end{array}$ \\
\hline $\begin{array}{l}\text { RT differences } \\
\text { between triplet } \\
\text { positions }\end{array}$ & $\begin{array}{l}H(2)=1.01 \\
\eta^{2}=0.0085\end{array}$ & $\begin{array}{l}\mathrm{H}(2)=1.37 \\
\eta^{2}=0.012\end{array}$ & $\begin{array}{l}H(2)=1.12 \\
\eta^{2}=0.0095\end{array}$ & $\begin{array}{l}H(2)=0.11, \\
\eta^{2}=0.00094\end{array}$ & $\begin{array}{l}H(2)=4.28 \\
\eta^{2}=0.077\end{array}$ \\
\hline Mean RTs by & $\left(1^{\mathrm{st}}\right) 424.6$ & $\left(1^{\mathrm{st}}\right) 412.2$ & $\left(1^{\text {st }}\right) 414.8$ & $\left(1^{\text {st }}\right) 394.2$ & $\left(1^{\text {st }}\right) 411.5$ \\
\hline triplet position & $\left(2^{\text {nd }}\right) 424.9$ & $\left(2^{\text {nd }}\right) 412.8$ & $\left(2^{\text {nd }}\right) 416.1$ & $\left(2^{\text {nd }}\right) 394.7$ & $\left(2^{\text {nd }}\right) 422.9$ \\
\hline (ms) & $\left(^{\text {rd }}\right) 424.2$ & $\left(3^{\text {rd }}\right) 409.1$ & $\left(3^{\text {rd }}\right) 416.5$ & $\left(3^{\text {rd }}\right) 395.5$ & $\left(3^{\text {rd }}\right) 421.7$ \\
\hline $\begin{array}{l}\text { Mean RTF1-2 } \\
(\mathrm{ms})+95 \% \mathrm{Cl}\end{array}$ & $\begin{array}{l}-0.27 ; \\
{[-0.94,0.40]}\end{array}$ & $\begin{array}{l}-0.60 ; \\
{[-1.17,0.015]}\end{array}$ & $\begin{array}{l}-0.86 ; \\
{[-1.46,-0.19]}\end{array}$ & $\begin{array}{l}-0.70 ; \\
{[-1.25,-0.11]}\end{array}$ & $\begin{array}{l}-11.8 ; \\
{[-13.2,-10.3]}\end{array}$ \\
\hline $\begin{array}{l}\text { Mean RTF1-3 } \\
(\mathrm{ms})+95 \% \mathrm{Cl}\end{array}$ & $\begin{array}{l}0.23 ; \\
{[-0.41,0.96]}\end{array}$ & $\begin{array}{l}3.19, \mathrm{p}<0.001 \\
{[2.55,3.93]}\end{array}$ & $\begin{array}{l}-1.09 ; \\
{[-1.80,-0.30]}\end{array}$ & $\begin{array}{l}-1.58 \\
{[-2.06,-1.12]}\end{array}$ & $\begin{array}{l}-10.7 ; \\
{[-12.3,-9.0]}\end{array}$ \\
\hline $\begin{array}{l}\text { Mean \% correct } \\
(2 \mathrm{AFC})+95 \% \mathrm{Cl}\end{array}$ & $\begin{array}{l}51.4, p=0.046 \\
{[51.2,51.6]}\end{array}$ & $\begin{array}{l}50.2 ; \\
{[50.0,50.4]}\end{array}$ & $\begin{array}{l}51.7, p=0.018 \\
{[51.5,51.9]}\end{array}$ & $\begin{array}{l}52.2 \\
p=0.0029 \\
{[52.0,52.4]}\end{array}$ & $\begin{array}{l}81.3, p<0.001 \\
{[80.6,82.0]}\end{array}$ \\
\hline $\begin{array}{l}\text { Mean triplets } \\
\text { created }\end{array}$ & $\begin{array}{l}0.15, p<0.001 \\
{[0.14,0.16]}\end{array}$ & $\begin{array}{l}0.13, p<0.001 \\
{[0.13,0.14]}\end{array}$ & $\begin{array}{l}0.15, p<0.001 \\
{[0.14,0.16]}\end{array}$ & $\begin{array}{l}0.17, p<0.001 \\
{[0.16,0.17]}\end{array}$ & $\begin{array}{l}3.30, p<0.001 ; \\
{[3.25,3.34]}\end{array}$ \\
\hline $\begin{array}{l}\text { Mean foils } \\
\text { created }\end{array}$ & $\begin{array}{l}0.083, p<0.001 ; \\
{[0.076,0.090]}\end{array}$ & $\begin{array}{l}0.083, p<0.001 \\
{[0.078,0.088]}\end{array}$ & $\begin{array}{l}0.075, p<0.001 ; \\
{[0.070,0.080]}\end{array}$ & $\begin{array}{l}0.092 \\
p<0.001 \\
{[0.087,0.097]}\end{array}$ & $\begin{array}{l}0.019 ; \\
{[0.014,0.023]}\end{array}$ \\
\hline $\begin{array}{l}\text { Difference } \\
\text { between foils and } \\
\text { real triplets } \\
\text { created }\end{array}$ & $\begin{array}{l}W=46.5 \\
M P R B C=-0.069\end{array}$ & $\begin{array}{l}W=87.5 \\
M P R B C=-0.081\end{array}$ & $\begin{array}{l}W=66.0 \\
M P R B C=-0.055\end{array}$ & $\begin{array}{l}W=73.5 \\
M P R B C=-0.059\end{array}$ & $\begin{array}{l}W=0.0 \\
p<0.001 \\
M P R B C=-0.94\end{array}$ \\
\hline $\begin{array}{l}\text { Spearman rho } \\
\text { (2AFC \& Creation) }\end{array}$ & 0.15 & $0.19, p=0.035$ & $0.20, p=0.026$ & $0.27, p=0.0029$ & 0.016 \\
\hline $\begin{array}{l}\text { Pearson r (RTF1-3 } \\
\text { \& 2AFC) }\end{array}$ & 0.046 & -0.046 & -0.12 & 0.034 & 0.042 \\
\hline $\begin{array}{l}\text { Spearman rho } \\
\text { (RTF1-3 \& } \\
\text { Creation) }\end{array}$ & 0.053 & -0.037 & -0.0060 & -0.014 & 0.068 \\
\hline
\end{tabular}

Notes. H statistics are calculated with Kruskal-Wallis H tests between groups (see "Effect Size Analyses"). Eta

8 squared $\left(\eta^{2}\right)$ is calculated following Equation 1. W statistics are calculated with Wilcoxon signed-rank tests (see

9 "Effect Size Analyses"). Matched pairs rank biserial correlation (MPRBC) is a measure of effect size (see "Effect Size

10 Analyses"). 
A

$$
\begin{gathered}
\text { RTs by } \\
\text { Triplet Position }
\end{gathered}
$$

B 2AFC Performance

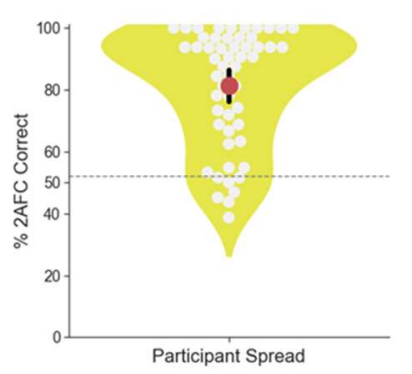

C Triplet Creation

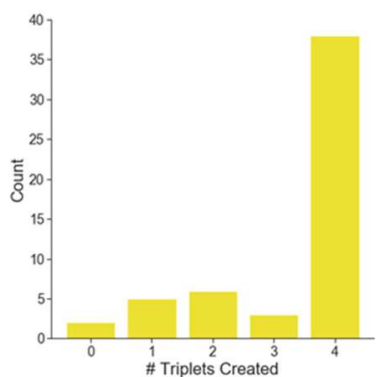

D Comparing Direct Measures

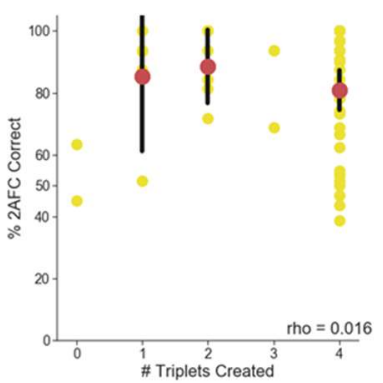

Figure 3. Results from Experiment 3 (Explicit Instruction). Performance across measures of statistical learning and correlation between direct measures. (A) Mean RTs by triplet position. Vertical yellow line segments indicate $95 \%$

Cls, computed across subjects. (B) Percentage of "real" triplets successfully chosen during 2AFC task. Gray dots indicate a single participant. Dashed gray line indicates the critical threshold for above-chance performance (alpha=0.05). (C) Histogram of the number of participants who created 0, 1, 2, 3 or 4 triplets successfully. (D) Spearman correlation between direct measures (2AFC \& Creation). Yellow dots represent individual participants. Red dots indicate means (when more than four data points are available). Black line segments indicate 95\% Cls.

Follow-Up Analysis: "Learners" show no RT facilitation. Participants in this experiment learned the triplet regularities (performing near ceiling on the 2AFC task) but showed no RT facilitation. Could the RT and 2AFC tests be measuring entirely distinct aspects of sequence learning? To test this idea in a setting without any explicit instruction, we checked whether successful "learners" from our previous (incidental exposure) experiments showed any RT facilitation. We found no statistically-significant evidence of RT facilitation even amongst the participants from Experiments $1 \& 2$ whose 2AFC performance was well above chance (all $H(2)<2.6$; all $\eta^{2}<0.022$ in all conditions for participants with $2 A F C$ accuracy $>=75 \%$; Supp. Fig. 4).

This lack of RT facilitation was also true of participants in Experiment $3\left(H(2)=4.47, \eta^{2}=0.081\right)$. 
1 Thus, regardless of whether participants were given incidental exposure (Exps. 1 \& 2) or explicit

2 instruction (Exp. 3), successful performance in 2AFC did not predict any response time speeding.

4 Discussion

We found that participants learned the triplet structure extremely well (approaching

6 ceiling performance in the $2 \mathrm{AFC}$ and Creation tasks) when provided with explicit instruction (Figs.

$73 \mathrm{~B} ; 3 \mathrm{C})$. This provided a positive control for our 2AFC and Creation testing paradigms. Thus, the

8 very weak evidence of learning in the direct measures in Experiments 1 \& 2 most likely reflect

9 properties of the incidental exposure method that was used in those traditional VSL experiments.

10 Explicit knowledge of triplet structure did not lead to response time facilitation (Fig. 3A).

11 We confirmed with informal in-lab tests that participants could theoretically use explicit

12 knowledge of the sequences to respond more quickly to targets, producing very large ( $>100 \mathrm{~ms})$

13 response time speeding for the target item. However, we observed no evidence of any

14 participants pursuing such a strategy. Thus, our response time facilitation measure was largely 15 insensitive to explicit knowledge of the triplet regularities.

\section{The stream position effect}

After testing more than 500 participants, we had found scant evidence of RT facilitation

20 in the TD task. Given the many positive effects previously reported for the TD task (e.g. Turk-

21 Browne et al., 2005; Kim et al., 2009; Campbell et al., 2012; Musz et al., 2015), we began to

22 question why we observed consistent null effects. We found that one of the differences between 
our TD task and prior experiments is that we ensured there was no relationship between the dependent variable of interest (triplet position) and the position of targets within each TD test stream (stream position). We counterbalanced the TD trials such that each image (regardless of triplet position) occurred equally often as all other images in each stream position (position 4, 5, 6, 7 or 8) (Fig. 5, top). However, in previous experiments using a TD task, this was not the case: each test stream would begin with an image from triplet position 1, so that later items in a triplet would occur (on average) later in the test stream (Fig. 5, middle). Thus, triplet position and stream position were confounded in prior studies, but de-confounded in our design.

Could the stream position of a target affect the response time to that target, regardless of the target's triplet position? If so, then response time facilitation could arise independently from any learning of sequence structure. A post-hoc analysis of data from Experiments 1, 2, and 3, revealed that RTs decreased as a function of stream position (Fig. 4A). Specifically, response times decreased by an average of $18.1 \mathrm{~ms}$ across consecutive positions in TD streams (min: 16.8 ms/item, max $20.2 \mathrm{~ms} /$ item, see Supp. Table 1). Moreover, this RT speeding effect of stream position was observed regardless of triplet position (Fig. 4B). We quantitatively confirmed this effect using linear regression (see "Multiple linear regression models" in Supp. Methods), with response time as the dependent variable and triplet position and stream position as predictor variables. We observed a significant effect of stream position (coeff=-17.3ms, $\mathrm{Cl}=[-18.2,-16.4]$, $t=-37.4, p<0.001)$. Adding triplet position to this linear model did not explain significant additional variance in the response times (coeff $=0.52 \mathrm{~ms}, \mathrm{Cl}=[-1.10,2.14], \mathrm{t}=0.625)$. Thus, faster responses to later stream positions might be the true underlying cause of RT speeding by triplet position (i.e. RT facilitation). In previous work, faster responses to later triplet positions were thought to 
1 arise from statistical learning during exposure; however, the results of this analysis suggest that

2 learning is not necessary to explain this triplet position speeding. In the traditional TD test design,

3 later triplet positions are always shown later in a stream relative to triplet first positions: thus,

4 the stream position effect of faster response times to images later in the stream can explain RT

5 facilitation effects, without any need to invoke triplet learning.

6

7

A
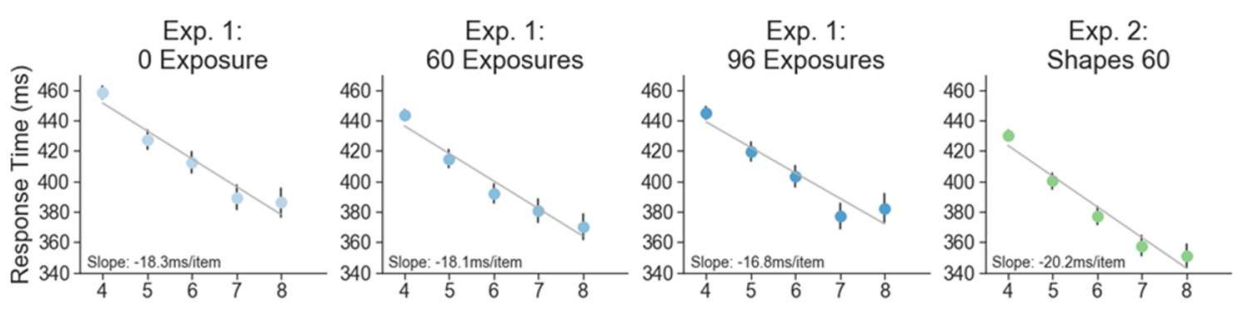

Exp. 2:

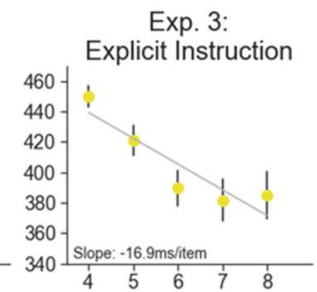

B
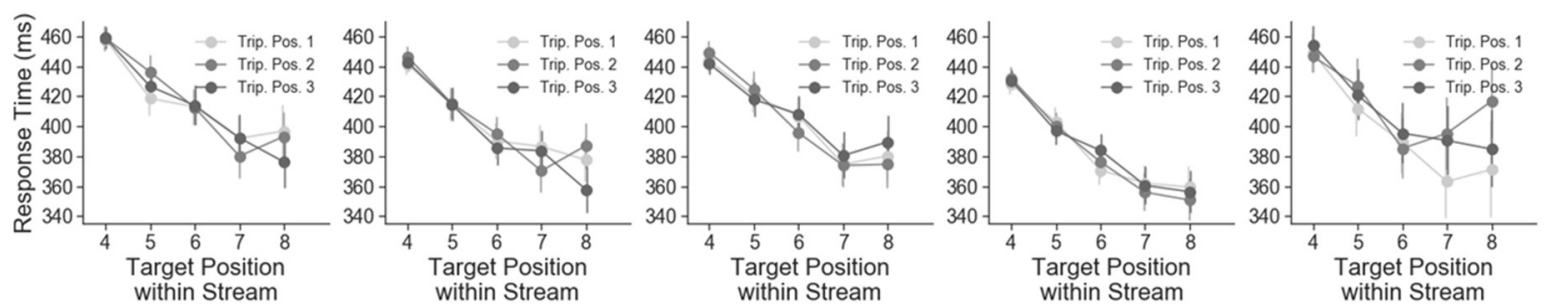

Figure 4. RTs by target stream position in the TD task. (A) RTs by stream position, collapsing across all triplet positions. Line of best fit shown in gray. (B) RTs by stream position, separated by triplet position. Shades of gray indicate data for a particular triplet position. Vertical line segments are 95\% Cls.

\section{Experiment 4}

Given that (i) TD stream position affects response time (Fig. 4), and (ii) prior experiments confounded stream position and triplet position in TD tasks, we set out to directly test whether the stream position confound could explain the response time facilitation reported in prior 
1 studies. We hypothesized that we could elicit RT facilitation by making a small change to the TD

2 task, while preserving the same stimuli and initial exposure phase from Experiment 1 (60

3 Exposures). To accomplish this, we intentionally introduced the same confound between stream

4 position and triplet position that is present in traditional TD tasks. Specifically, each TD trial: (i)

5 would begin with an image from triplet position 1, and (ii) would present each of the four triplets

6 exactly once, for a total of 12 images. If we observe RT facilitation using this "traditional" TD test,

7 then this would suggest that the properties of the traditional TD paradigm were indeed

8 contributing to the RT facilitation effects, rather than the statistical regularities in the initial

9 exposure phase.

10

\section{Method}

Participants. 120 participants (Supp. Table 1; Supp. Fig. 1) were analyzed from data

13 collected via an advertisement on Amazon's Mechanical Turk platform created using psiTurk

142.2 .3 (Gureckis et al., 2016). Participants received monetary compensation for completing the 15 experiment (Supp. Table 1).

Exclusion Criteria. 268 subjects participated, with 102 participants excluded for violating one of the exclusion criteria (Supp. Table 1) and 46 participants were left unanalyzed to ensure counterbalancing.

Stimuli. Identical to Experiment 1: 60 Exposures.

Procedure. Identical to Experiment 1: 60 Exposures, except for the TD Task.

21 We modified the TD task to better match the traditional testing method (e.g. Kim et al., 2009;

22 Otsuka et al., 2016). Thus, instead of having variable length trials (Fig. 5, top), each of the 72 trials 
1

consisted of exactly 12 elements (i.e. all four triplets on each trial; Fig. 5, middle). Targets never appeared in the first three or last three positions of the stream, and so there were 6 stream positions where the target could occur. Importantly, each trial begin with a triplet position 1 image (e.g. $C_{1}$ ) and ended with a triplet position 3 image (e.g. $D_{3}$ ). Crucially, this meant that targets were no longer balanced by stream position: first triplet position stimuli could only appear in stream positions 4 or 7 , second triplet position images could only appear in stream positions 5 or 8 , and third triplet position images could only appear in stream positions 6 or 9.

8

\section{Results}

As hypothesized, we observed a significant difference in RTs by triplet position $(H(2)=82.3$, $p<0.001, \eta^{2}=0.41$ ), as well as a monotonic decrease in response times by triplet position (Fig. $6 \mathrm{~A}$; Table 2). Bootstrap tests of RT facilitation against chance were highly significant (RTF1-3 mean=31.8ms, 95\% Cl=[31.1, 32.6], $\mathrm{p}<0.001$; Table 2). As in Experiments 1-3, RTs decreased as a function of stream position (Fig. 6D), by approximately $15 \mathrm{~ms}$ per stream position. Note that, although triplet position and stream position are confounded by design in this experiment, the data suggested that stream position was the primary driver of RT facilitation: stream position 5 (the first item in a triplet) was detected more quickly than stream position 4 (the final item in a triplet) (Fig. 6E). Thus, we observed robust RT facilitation, and it was likely driven by the stream position effect, rather than learning of triplet sequences.

As in Experiments 1 \& 2, there was weak, but statistically significant, evidence of learning in both direct measures (2AFC: mean $=51.9 \%, 95 \% \mathrm{Cl}=[51.7,52.1], \mathrm{p}<0.01$, Fig. $6 \mathrm{~B}$; Creation: mean $=0.24,95 \% \mathrm{Cl}=[0.23,0.25], \mathrm{p}<0.001$, Fig. 6C; Table 2). Real triplets were created at more 
1 than twice the rate of foils (Foil Creation: mean=0.11, 95\% $\mathrm{Cl}=[0.10,0.11], \mathrm{p}<0.001$; Supp. Fig. 2),

2 and this difference was near our statistical threshold ( $W=125, p=0.056, M P R B C=-0.083)$, .

3 Moreover, there was again a positive and significant correlation between performance on the

4 direct tasks (2AFC \& Creation: Spearman rho $=0.305, p<0.001$, Fig. $6 F$ ). Finally, consistent with

5 findings from Experiments 1-3, there was no linear relationship between performance in the

6 indirect and direct tasks (RTF1-3 \& 2AFC: Pearson r=0.028; Supp. Fig. 3A; RTF1-3 \& Creation:

7 Spearman rho $=0.17, p=0.062$; Supp. Fig. 3B).

\section{Discussion} observed the typically reported RT facilitation effect (Fig. 6A). RT facilitation has been treated as evidence for implicit visual statistical learning, but our findings across four experiments (Figs. 2A; $3 A ; 4 ; 6 A)$, suggest that the previous evidence of RT facilitation is instead an artifact of the testing paradigm. Consistent with this interpretation, there was no significant correlation between performance on the TD task and either of the direct measures (Supp. Fig. 3).

Can the TD testing paradigm generate a response time facilitation effect, even when no statistical learning occurs? Our data so far suggest that this is the case, but they are not definitive. No response time facilitation was observed in Experiments 1, 2 and 3, when the stream position

19 confound was absent. However, when the stream position confound was present in Experiment

204 , we observed robust response time facilitation. Thus, it seems that the confounding of the

21 stream and triplet positions was the cause of the RT facilitation. However, another possibility is

22 that some learning was occurring in the initial exposure phase, and that this learning could only 
1 be expressed when the TD task was structured as in Experiment 4. To rule out this possibility, we

2 decided to run a final experiment in which we preserve the TD stream position confound, but

3 eliminate the statistical regularities, so that any statistical learning during the initial exposure will

4 be of no use in the TD task.

5

\section{Experiment 5}

We scrambled the regularities in the TD task to determine if the stream position confound

9 is sufficient to produce the RT facilitation effect when regularities are removed. We (i) kept the

10 initial exposure phase identical to Experiment 4, and (ii) kept the identity and position of each TD

11 target unchanged, but (iii) scrambled the sequence of all other images within each TD trial (Fig.

125 , bottom). In this way, the exposure phase was identical to prior experiments, and the TD targets

13 and stream positions were identical, but it was not possible for participants to leverage

14 regularities from the exposure phase to respond more rapidly in the target detection (TD) task.

15 If we still observe RT facilitation in this design, despite the absence of any sequential regularities

16 in the TD task, then this would be especially strong evidence that RT facilitation in TD tasks can

17 arise without any statistical learning at all.

19 Method

Participants. Identical to Experiment 4. 
Exclusion Criteria. 207 subjects participated, with 75 participants excluded for violating one of the exclusion criteria (Supp. Table 1) and 12 participants were left unanalyzed to ensure

3 counterbalancing.

Stimuli. Identical to Experiment 4.

Procedure. Identical to Experiment 4, except for the Target Detection (TD) Task. The stream position of targets in the TD task was preserved from Experiment 4, but the stream

7 position of the other 11 images in the stream was randomized (Fig. 5, bottom). This procedure

8 eliminated the triplet structure from the TD task. Thus, statistical learning during initial exposure could not lead to response time speeding of the later items in a triplet during the TD task. All

10 other phases of the experiment were identical to Experiment 4.
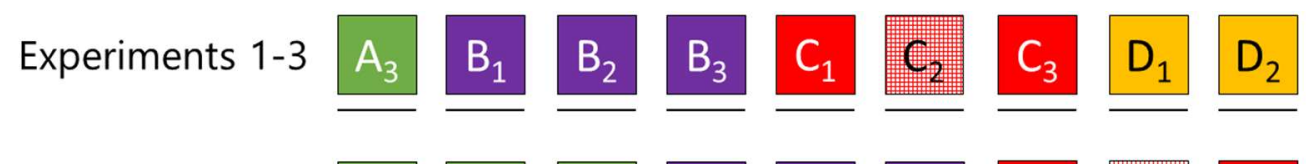

$$
\text { Experiment } 4
$$
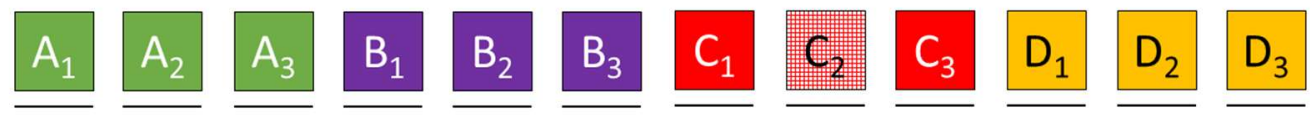

Experiment 5
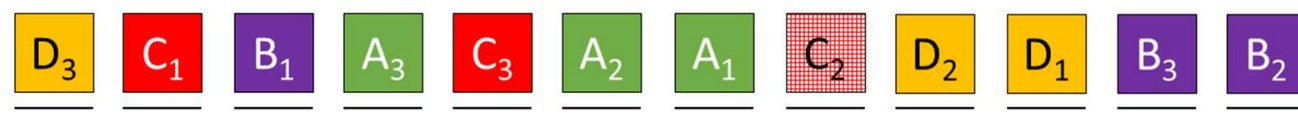

$$
\text { Stream Position } 12
$$

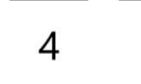

Figure 5. Example stimulus streams from the target detection task, indicating how stream structure varied across

14 (top row) Experiments 1-3, (middle row); Experiment 4, and Experiment 5 (bottom row). Stream position indicates

$$
\text { the ordinal position in which a stimulus appears. Background fill color indicates triplet membership. Subscript }
$$




\section{Results}

As in Experiment 4, we found that RTs differed significantly by triplet position $(H(2)=32.1$,

$3 p<0.001, \eta^{2}=0.22$ Fig. $\left.6 A\right)$. Furthermore, images in later triplet positions were again detected

4 more quickly than images in earlier triplet positions (RTF1-3 mean=22.2ms, $95 \% \mathrm{Cl}=[21.6,22.9]$,

$5 \quad \mathrm{p}<0.001 ;$ Table 2). As in all other experiments, RTs decreased monotonically as a function of

6 stream position (Fig. 6D). Thus, despite the absence of any triplet regularities in this version of

7 the TD task, the covariance of stream position and triplet position still gave the appearance of RT

8 facilitation between triplet positions (Fig. 6D; 6E; Table 2).

9 In the first of the two direct measures, 2AFC performance was no different than chance

10 (mean=48.9\%, Fig. 6B). Creation performance was greater than chance (mean=0.15, 95\%

$11 \mathrm{Cl}=[0.14,0.16], \mathrm{p}<0.001$, Fig. $6 \mathrm{C}$ ), but creation of foils (from the 2AFC) was also greater than

12 chance $(\operatorname{mean}=0.14,95 \% \mathrm{Cl}=[0.13,0.15], \mathrm{p}<0.001$; Supp. Fig. 2; Table 2). Real triplets were

13 created slightly more than foils, with no significant difference between triplets and foils created

$14(\mathrm{~W}=115, \mathrm{MPRBC}=-0.020$; Supp. Fig. 2). 

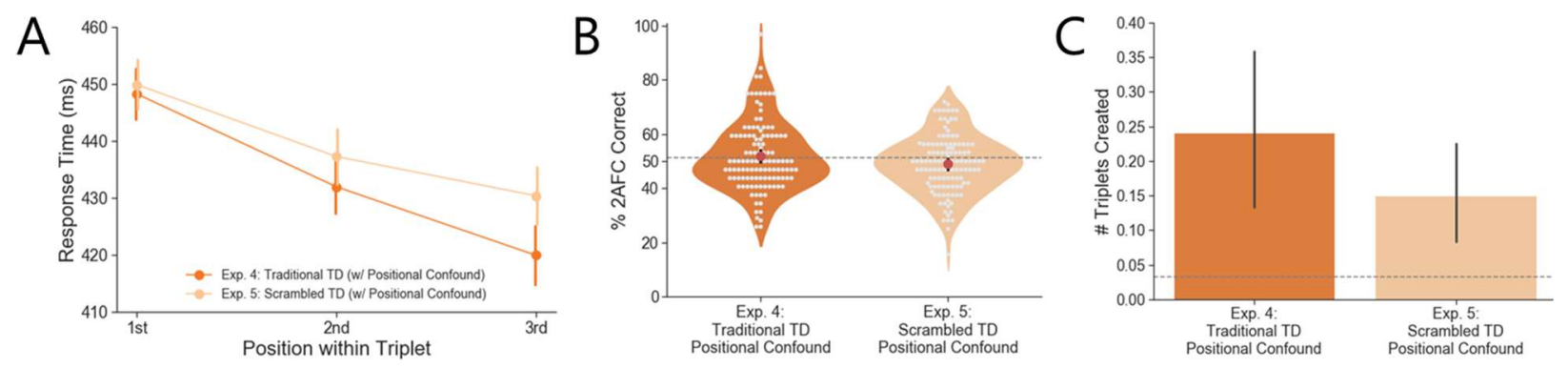

$\mathrm{D}$

Exp. 4: Traditional TD

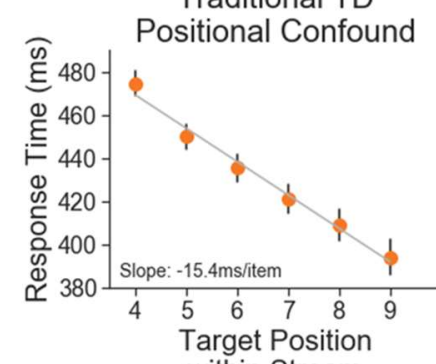
within Stream
Exp. 5:

Scrambled TD Positional Confound

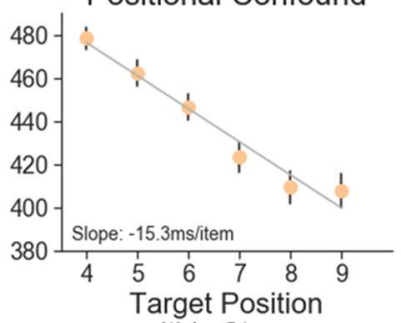

$\mathrm{E}$

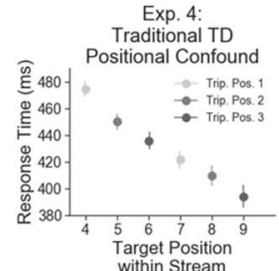

Exp. 5: Scrambled TD Positional Con

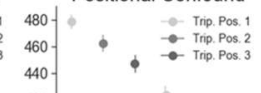

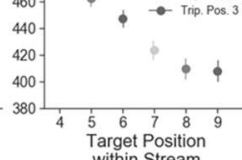

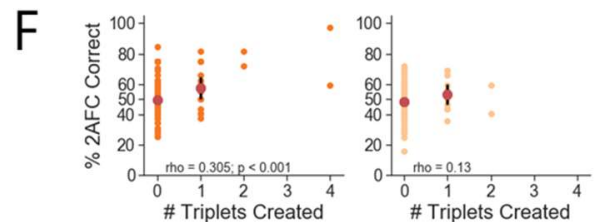

Figure 6. Indirect and direct measure performance in Experiments 4 \& 5. (A) Mean RTs by triplet position.

3 (B) Distribution of 2AFC accuracy across participants. Gray dots indicate the accuracy of a single participant. (C)

4 Mean number of triplets created successfully. Dashed line indicates critical threshold above chance (alpha $=0.05$ )

5 (D) RTs by stream position. Line of best fit also shown. (E) RTs by stream position when separating by triplet

6 position. (F) Spearman correlation between direct measures (2AFC \& Creation). Black line segments indicate 95\%

7 Cls. Dashed lines indicate chance performance (alpha $=0.05)$. Red dots indicate means.

9 Table 2

10 Results from Experiments 4 \& 5

\begin{tabular}{|c|c|c|}
\hline & Exp. 4 (Traditional TD) & Exp. 5 (Scrambled TD) \\
\hline RT difference across triplet positions & $H(2)=82.3, p<0.001, n^{2}=0.41$ & $H(2)=32.1, p<0.001, \eta^{2}=0.22$ \\
\hline Mean RTs by triplet position (ms) & $\begin{array}{l}\left(1^{\text {st }}\right) 448.2 \\
\left(2^{\text {nd }}\right) 432.0 \\
\left(3^{\text {rd }}\right) 419.9\end{array}$ & $\begin{array}{l}\left(1^{\text {st }}\right) 449.9 \\
\left(2^{\text {nd }}\right) 437.3 \\
\left(3^{\text {rd }}\right) 430.4\end{array}$ \\
\hline Mean RTF1-2 (ms) + 95\% Cl & $18.2, \mathrm{p}<0.001 ;[17.6,18.8]$ & $13.6, p<0.001 ;[13.1,14.2]$ \\
\hline Mean RTF1-3 (ms) + 95\% Cl & $31.8, p<0.001 ;[31.1,32.6]$ & $22.2, p<0.001 ;[21.6,22.9]$ \\
\hline Mean $\%$ correct $(2 \mathrm{AFC})+95 \% \mathrm{Cl}$ & $51.9, p<0.01 ;[51.7,52.1]$ & 48.9; [48.7, 49.1] \\
\hline Mean triplets created & $0.24, p<0.001 ;[0.23,0.25]$ & $0.15, \mathrm{p}<0.001 ;[0.14,0.16]$ \\
\hline
\end{tabular}




$\begin{array}{lll}\text { Mean foils created } & 0.11, \mathrm{p}<0.001 ;[0.10,0.11] & 0.14, \mathrm{p}<0.001 ;[0.13,0.15] \\ \begin{array}{lll}\text { Difference between foils and real } \\ \text { triplets created }\end{array} & \begin{array}{l}\mathrm{W}=125.0, \mathrm{p}=0.056, \\ \mathrm{MPRBC}=-0.083\end{array} & \mathrm{~W}=115.0, \\ \text { Spearman rho (2AFC \& Creation) } & 0.31, \mathrm{p}<0.001 & \mathrm{MPRBC}=-0.020 \\ \text { Pearson r (RTF1-3 \& 2AFC) } & 0.028 & 0.13 \\ & & 0.096 \\ \text { Spearman rho (RTF1-3 \& Creation) } & 0.17 & 0.035\end{array}$

1

17

Notes. H statistics are calculated with Kruskal-Wallis H tests between groups (see "Effect Size Analyses"). Eta squared $\left(\eta^{2}\right)$ is calculated following Equation 1. W statistics are calculated with Wilcoxon signed-rank tests (see "Effect Size Analyses"). Matched pairs rank biserial correlation (MPRBC) is a measure of effect size (see "Effect Size Analyses").

There was no significant relationship between the direct measures, though, numerically the correlation was (as in all experiments) again positive (2AFC \& Creation: Spearman rho=0.13; Fig. 6F). As in all earlier experiments, there was no significant relationship between the indirect and direct measures of VSL (RTF1-3 \& 2AFC: Pearson r=0.096; Supp. Fig. 3A; RTF1-3 \& Creation: Spearman rho=0.035; Supp. Fig. 3B).

Follow-Up Analysis: Learning During Test. Might learning have occurred during the TD task itself, rather than during the incidental exposure phase that preceded the TD task? We consider this issue in detail in Supplemental Material ("Learning During Test"). These analyses indicate that some forms of learning are indeed occurring during the traditional TD Test, which may inform the design of future VSL experiments employing target detection tasks.

\section{Discussion} As hypothesized, we observed response time facilitation in the TD task, even when the ordering of items in the test stream was randomized. Thus, it appears that the stream position 
1 effect is a sufficient explanation for response time facilitation, producing facilitation even when

2 learning the triplet regularities could never facilitate the responses (because they were never

3 present in the first place). In combination with our other results, this suggests that prior

4 experiments employing the TD paradigm to measure visual statistical learning cannot be taken

5 as evidence of implicit statistical learning. Instead, the regularities in the exposure phase appear

6 to have no effect on response time facilitation (Figs. 2B; 3A).

\section{General Discussion}

We examined the automaticity of statistical learning by exposing participants to visual

11 triplet sequences and comparing their performance across different exposure conditions and

12 measures of learning. Under conditions of passive exposure, participants unexpectedly

13 demonstrated no evidence of learning using response time facilitation as a dependent measure,

14 and they exhibited very weak evidence of learning using two direct measures of learning

15 (Experiments 1 \& 2). We then explicitly informed participants about the triplet regularities, which

16 greatly improved performance on the direct measures, but did not improve performance in the

17 indirect response time measure (Experiment 3). Finally, we demonstrated that previous evidence

18 for visual statistical learning from target detection response times may be flawed, due to a

19 confound with test stream positions (Experiments $4 \& 5$ ). Practically, these results suggest that

20 great care should be taken in designing response time tests for statistical learning and in selecting

21 the method of exposure to regularities. Conceptually, the data indicate that visual statistical 
1 learning is not an automatic and passive learning process. Instead, learning appears to requires

2 a more active form of engagement with sequential regularities.

3

4 Does statistical learning arise from passive exposure to visual regularities?

6 intentionally employed a perceptual cover task, reducing the possibility that participants would

7 explicitly attend to the relationships between images (Turk-Browne et al., 2009) in order to keep

8 the learning passive and incidental (Aslin, 2017). However, more active forms of exposure are

9 widely used in the literature. One form of active exposure is to associate specific actions or

10 category decisions with the regularities to be learned (e.g. Nissen \& Bullemer, 1987; Jiménez \&

11 Méndez, 1999; Conway \& Christiansen, 2005; Turk-Browne et al., 2010). Similarly, another active

12 form of exposure is to encourage relational processing between stimuli. For example, in a 1-back

13 task, participants must compare each stimulus to the preceding one, potentially drawing

14 attention to relationships between stimuli (Turk-Browne et al., 2005; Brady \& Oliva, 2008; Arciuli

15 \& Simpson, 2012; Campbell et al., 2012; Stevens, Arciuli, \& Anderson, 2014; Musz et al., 2015;

16 Vickery, Park, Gupta, \& Berryhill, 2018)

19 does not arise from passive exposure to visual regularities. Incidental exposure to visual

20 regularities did not produce faster response times to predictable images (Fig. 2A), nor did it

21 produce any robust evidence of learning in direct measures (Fig. 2B; 2C). Contrary to our pre- 
1 registered hypotheses (Exp. 1), the indirect measure never provided evidence for learning, even

2 when participants had explicit knowledge of the regularities (Fig. 3A).

3 Although performance in some measures of VSL is known to be influenced by explicit

4 knowledge (Bertels et al., 2015), the core learning processes have been conceived of as arising

5 without requiring explicit knowledge (Perruchet \& Pacton, 2006). Indeed, statistical learning is

6 often described as an unconscious (Turk-Browne et al., 2010; Turk-Browne, 2012) or incidental

7 (Saffran et al., 1997) cognitive process. However, we only observed robust learning when

8 participants were actively seeking to discover the triplet regularities (Exp. 3). Under these

9 conditions, most participants achieved ceiling performance $(>75 \%$ on the 2 AFC task and re-

10 creating all four triplets) after fewer than 4 rounds of presentation and test on the triplet

11 structure (Fig. 3B; 3C). Additionally, across all experiments, our two direct measures of VSL were

12 consistently positively correlated (Tables 1 \& 2): participants who expressed greater "familiarity"

13 with the image regularities (assessed via 2AFC) could also explicitly create more of the sequence

14 structures. Altogether, these results suggest that passive attention is not sufficient for learning:

15 whenever we did observe successful VSL, it emerged from active engagement with the

16 regularities (Exp. 3) and was associated with explicit knowledge (Creation performance).

17 We were surprised at the weak evidence of learning in the direct measures: in the 2AFC

18 task, forced-choice performance was below 52\%, and was no better with 96 exposures than with

190 exposures during the passive cover task (Fig. 2). Some statistical learning studies with passive

20 exposure to visual regularities have produced 2AFC performance as high as 95\% (Fiser \& Aslin,

21 2002). However, typical 2AFC performance in VSL studies ranges from chance to approximately

22 75\% correct (e.g. Arciuli \& Simpson, 2012; Arciuli, Torkildsen, Stevens, \& Simpson, 2014; Otsuka 
et al., 2016; Turk-Browne, Scholl, Chun, \& Johnson, 2009; c.f. Schlichting et al., 2017), even when participants had previously participated in a statistical learning experiment (Bogaerts, Siegelman,

3 \& Frost, 2016). One might suppose that the weak 2AFC performance in our passive exposure

4 experiments could reflect a lack of attention to the stimuli. However, it is unlikely that participants in our experiments performed poorly due to lack of attention: we stringently selected participants for task compliance; they detected targets rapidly (mean RTs < 460ms) and accurately (cover task performance >=90\%) in all experiments, both during exposure and during test (Supp. Table 1); and the participant pool is clearly able to learn, as shown in Experiment 3 . The most likely explanation of the poor 2AFC performance in Experiments 1 and 2 arises from the method of exposure to the triplet regularities: the demands of our cover task did not require

11 the participants to deeply process the stimuli or to associate the features of consecutive stimuli. Although we cannot conclusively determine which processes are necessary for visual statistical learning, it appears that passive attention is not sufficient on its own.

Should the target detection task be used to assess learning?

Indirect measures of VSL, such as the target detection (TD) task, are considered to provide

17 the clearest evidence of implicit knowledge of incidentally learned regularities. Direct measures of VSL, such as 2AFC or Creation tasks, may draw on more explicit knowledge (Turk-Browne et al., 2005; Bertels et al., 2015; Batterink, Reber, Neville, et al., 2015). The TD paradigm has been used to investigate the dependence of VSL on attention (Turk-Browne et al., 2005; Musz et al.,

21 2015), the permanence of knowledge acquired via VSL (Kim et al., 2009), how VSL is affected in 22 aging (Campbell et al., 2012), and the underlying neural correlates of VSL (Otsuka \& Saiki, 2017). 
1 We suggest that these conclusions be reviewed in light of the stream position effect. For example,

2 previous work has suggested that visual statistical learning is implicit and long-lasting (>24 hours)

3 (Kim et al., 2009). However, given that stream position confounds are sufficient to produce the

4 speeding effects from prior experiments (Exps. 4 \& 5), these conclusions need to be revisited. An important design decision in implementations of the TD task is the length of the test stream. Our stream length was limited to ensure there were no repetitions of images in any TD

7 stream: if a target repeats within (say) 30 seconds, then speeding of the response to that target 8 may be due to short-term priming of items or associations, rather than a learning effect that will 9 persist in long-term memory. We present further considerations of stream length in 10 Supplemental Material ( "Stream Length in Target Detection Tasks").

\section{Multiple components of statistical learning}

Statistical learning is now thought to comprise multiple cognitive processes (Bays et al.,

14 2015; Arciuli, 2017; Batterink, Paller, \& Reber, 2019), which may be engaged similarly (Kirkham et al., 2002; Perruchet \& Pacton, 2006) or differently (Conway \& Christiansen, 2005; Frost et al., 2015; Siegelman \& Frost, 2015) across sensory modalities. Consistent with a multiple cognitive

17 process view, we found that indirect and direct measures of learning were not significantly correlated in any of our experiments (Supp. Fig. 3B), even when we explicitly instructed

19 participants to learn. Consistent with the idea that statistical learning can differ across modalities,

20 we found no evidence of visual statistical learning using the indirect response time task (Figure

21 2), even while a similar task has demonstrated rapid (if transient) statistical learning in auditory 22 paradigms (Batterink, 2017). 


\section{Additional Considerations}

The learning processes involved in any experiment may vary according to the cognitive

4 demands of the exposure phase, the familiarity and complexity of stimulus sets, the

5 developmental stage of participants, and the sensory modality of stimulus presentation (Craik \&

6 Lockhart, 1972; Siegelman et al., 2018; Arciuli, 2017; Arciuli \& Conway, 2018; Frost et al., 2015).

7 In this context, it makes sense to exercise caution in generalizing results from one experiment to

8 another. In addition, the details of experimental design and data processing become especially

9 important for interpretation; we echo calls for documentation and sharing of experiments and

10 their data (e.g. Stall et al., 2019). Our pre-registered experiments, code and data are available at

11 https://osf.io/vtmpb.

12 It will be important to determine how the subcomponents of statistical learning are

13 related to established cognitive processes, such as associative learning (which may be less

14 automatic) and motor learning (which may be more automatic). Neurally, there is evidence that

15 many cortical and subcortical regions are involved in statistical learning (Finn, Kharitonova,

16 Holtby, \& Sheridan, 2018). Specifically, that medial temporal lobe regions might be necessary for

17 VSL (Schapiro et al., 2014, 2012; c.f. Covington, Brown-Schmidt, \& Duff, 2018). But if VSL was

18 measured using a direct forced-choice recognition measure in these studies (potentially drawing

19 on explicit associations) the necessity of MTL regions for this task may be less surprising. Going

20 forward, it seems important to specify subtypes of statistical learning, and to test whether these

21 subtypes are supported by distinct neural processes. 


\section{Conclusion}

In summary, we found weak evidence of learning using direct measures of visual statistical learning, and no evidence of learning using a common reaction time measure. Participants who recognized visual sequence regularities in a forced-choice task could also often recreate the sequences when explicitly probed, indicating their knowledge was not entirely implicit. Altogether, the data suggest that visual statistical learning does not occur automatically, even when attention is directed to the stimuli. Rather, it appears that some further active engagement with stimulus properties is required to extract sequential regularities.

\section{References}

Arciuli, J. (2017). The multi-component nature of statistical learning. Phil. Trans. R. Soc. B, 372(1711), 20160058. https://doi.org/10.1098/rstb.2016.0058

Arciuli, J., \& Conway, C. M. (2018). The Promise-And Challenge-Of Statistical Learning for Elucidating Atypical Language Development. Current Directions in Psychological Science, 0963721418779977. https://doi.org/10.1177/0963721418779977

Arciuli, J., \& Simpson, I. C. (2012). Statistical learning is lasting and consistent over time. Neuroscience Letters, 517(2), 133-135. https://doi.org/10.1016/j.neulet.2012.04.045

Arciuli, J., Torkildsen, J. von K., Stevens, D. J., \& Simpson, I. C. (2014). Statistical learning under incidental versus intentional conditions. Frontiers in Psychology, 5. https://doi.org/10.3389/fpsyg.2014.00747 
Aslin, R. N. (2017). Statistical learning: A powerful mechanism that operates by mere exposure. Wiley Interdisciplinary Reviews: Cognitive Science, 8(1-2), e1373. https://doi.org/10.1002/wcs.1373

Barakat, B. K., Seitz, A. R., \& Shams, L. (2013). The effect of statistical learning on internal stimulus representations: Predictable items are enhanced even when not predicted. Cognition, 129(2), 205-211. https://doi.org/10.1016/j.cognition.2013.07.003

Batterink, L. J. (2017). Rapid Statistical Learning Supporting Word Extraction From Continuous Speech. Psychological Science, 0956797617698226. https://doi.org/10.1177/0956797617698226

Batterink, L. J., Paller, K. A., \& Reber, P. J. (2019). Understanding the Neural Bases of Implicit and Statistical Learning. Topics in Cognitive Science, 11(3), 482-503. https://doi.org/10.1111/tops.12420

Batterink, L. J., Reber, P. J., Neville, H. J., \& Paller, K. A. (2015). Implicit and explicit contributions to statistical learning. Journal of Memory and Language, 83, 62-78. https://doi.org/10.1016/j.jml.2015.04.004

Batterink, L. J., Reber, P. J., \& Paller, K. A. (2015). Functional differences between statistical learning with and without explicit training. Learning \& Memory, 22(11), 544-556. https://doi.org/10.1101/lm.037986.114

Bays, B. C., Turk-Browne, N. B., \& Seitz, A. R. (2015). Dissociable behavioural outcomes of visual statistical learning. Visual Cognition, 23(9-10), 1072-1097. https://doi.org/10.1080/13506285.2016.1139647

Bertels, J., Destrebecqz, A., \& Franco, A. (2015). Interacting Effects of Instructions and Presentation Rate on Visual Statistical Learning. Frontiers in Psychology, 6. https://doi.org/10.3389/fpsyg.2015.01806 
1 Bertels, J., Franco, A., \& Destrebecqz, A. (2012). How implicit is visual statistical learning? Journal of Experimental Psychology: Learning, Memory, and Cognition, 38(5), 14251431. https://doi.org/10.1037/a0027210

Bogaerts, L., Siegelman, N., \& Frost, R. (2016). Splitting the variance of statistical learning performance: A parametric investigation of exposure duration and transitional probabilities. Psychonomic Bulletin \& Review, 23(4), 1250-1256. https://doi.org/10.3758/s13423-015-0996-z

Brady, T. F., \& Oliva, A. (2008). Statistical Learning Using Real-World Scenes: Extracting Categorical Regularities Without Conscious Intent. Psychological Science, 19(7), 678685. https://doi.org/10.1111/j.1467-9280.2008.02142.x

Campbell, K. L., Zimerman, S., Healey, M. K., Lee, M. M. S., \& Hasher, L. (2012). Age differences in visual statistical learning. Psychology and Aging, 27(3), 650-656. https://doi.org/10.1037/a0026780

Conway, C. M., \& Christiansen, M. H. (2005). Modality-Constrained Statistical Learning of Tactile, Visual, and Auditory Sequences. Journal of Experimental Psychology: Learning, Memory, and Cognition, 31(1), 24-39. https://doi.org/10.1037/0278-7393.31.1.24

Covington, N. V., Brown-Schmidt, S., \& Duff, M. C. (2018). The Necessity of the Hippocampus for Statistical Learning. Journal of Cognitive Neuroscience, 30(5), 680-697. https://doi.org/10.1162/jocn_a_01228

Craik, F. I. M., \& Lockhart, R. S. (1972). Levels of processing: A framework for memory research. Journal of Verbal Learning and Verbal Behavior, 11(6), 671-684. https://doi.org/10.1016/S0022-5371(72)80001-X

Craik, F. I. M., \& Tulving, E. (1975). Depth of processing and the retention of words in episodic memory. Journal of Experimental Psychology: General, 104(3), 268-294. https://doi.org/10.1037/0096-3445.104.3.268 
1 Fernandes, T., Kolinsky, R., \& Ventura, P. (2010). The impact of attention load on the use of statistical information and coarticulation as speech segmentation cues. Attention, Perception, \& Psychophysics, 72(6), 1522-1532. https://doi.org/10.3758/APP.72.6.1522

4 Finn, A. S., \& Hudson Kam, C. L. (2008). The curse of knowledge: First language knowledge impairs adult learners' use of novel statistics for word segmentation. Cognition, 108(2), 477-499. https://doi.org/10.1016/j.cognition.2008.04.002

Fiser, J., \& Aslin, R. N. (2002). Statistical learning of higher-order temporal structure from visual shape sequences. Journal of Experimental Psychology: Learning, Memory, and Cognition, 28(3), 458-467. https://doi.org/10.1037/0278-7393.28.3.458

Fiser, J., \& Aslin, R. N. (2005). Encoding Multielement Scenes: Statistical Learning of Visual Feature Hierarchies. Journal of Experimental Psychology: General, 134(4), 521-537. https://doi.org/10.1037/0096-3445.134.4.521

Fiser, J., Scholl, B. J., \& Aslin, R. N. (2007). Perceived object trajectories during occlusion constrain visual statistical learning. Psychonomic Bulletin \& Review, 14(1), 173-178. https://doi.org/10.3758/BF03194046

Frost, R., Armstrong, B. C., Siegelman, N., \& Christiansen, M. H. (2015). Domain generality versus modality specificity: The paradox of statistical learning. Trends in Cognitive Sciences, 19(3), 117-125. https://doi.org/10.1016/j.tics.2014.12.010 
1 Glicksohn, A., \& Cohen, A. (2011). The role of Gestalt grouping principles in visual statistical learning. Attention, Perception, \& Psychophysics, 73(3), 708-713. https://doi.org/10.3758/s13414-010-0084-4

Gureckis, T. M., Martin, J., McDonnell, J., Rich, A. S., Markant, D., Coenen, A., ... Chan, P. (2016). psiTurk: An open-source framework for conducting replicable behavioral experiments online. Behavior Research Methods, 48(3), 829-842. https://doi.org/10.3758/s13428-015-0642-8

Jiménez, L., \& Méndez, C. (1999). Which attention is needed for implicit sequence learning? Journal of Experimental Psychology: Learning, Memory, and Cognition, 25(1), 236-259. https://doi.org/10.1037/0278-7393.25.1.236

Jones, E., Oliphant, T., Peterson, P., \& others. (2001). SciPy: Open source scientific tools for Python. Retrieved from http://www.scipy.org

Kerby, D. S. (2014). The Simple Difference Formula: An Approach to Teaching Nonparametric Correlation. Comprehensive Psychology, 3, 11.IT.3.1. https://doi.org/10.2466/11.IT.3.1

Kim, R., Seitz, A., Feenstra, H., \& Shams, L. (2009). Testing assumptions of statistical learning: Is it long-term and implicit? Neuroscience Letters, 461(2), 145-149. https://doi.org/10.1016/j.neulet.2009.06.030

Kirkham, N. Z., Slemmer, J. A., \& Johnson, S. P. (2002). Visual statistical learning in infancy: Evidence for a domain general learning mechanism. Cognition, 83(2), B35-B42. https://doi.org/10.1016/S0010-0277(02)00004-5

Kraut, R., Olson, J., Banaji, M., Bruckman, A., Cohen, J., \& Couper, M. (2004). Psychological Research Online: Report of Board of Scientific Affairs' Advisory Group on the Conduct of Research on the Internet. American Psychologist, 59(2), 105-117. https://doi.org/10.1037/0003-066X.59.2.105 
1 Murphy, K. R., Myors, B., \& Wolach, A. (2014). Statistical Power Analysis: A Simple and

General Model for Traditional and Modern Hypothesis Tests, Fourth Edition. https://doi.org/10.4324/9781315773155

Musz, E., Weber, M. J., \& Thompson-Schill, S. L. (2015). Visual statistical learning is not reliably modulated by selective attention to isolated events. Attention, Perception, \& Psychophysics, 77(1), 78-96. https://doi.org/10.3758/s13414-014-0757-5

Nissen, M. J., \& Bullemer, P. (1987). Attentional requirements of learning: Evidence from performance measures. Cognitive Psychology, 19(1), 1-32. https://doi.org/10.1016/0010-0285(87)90002-8

Olson, I. R., \& Chun, M. M. (2001). Temporal contextual cuing of visual attention. Journal of Experimental Psychology: Learning, Memory, and Cognition, 27(5), 1299-1313. https://doi.org/10.1037/0278-7393.27.5.1299

Orbán, G., Fiser, J., Aslin, R. N., \& Lengyel, M. (2008). Bayesian learning of visual chunks by human observers. Proceedings of the National Academy of Sciences, 105(7), 27452750. https://doi.org/10.1073/pnas.0708424105

Otsuka, S., Koch, C., \& Saiki, J. (2016). Visual statistical learning produces implicit and explicit knowledge about temporal order information and scene chunks: Evidence from direct and indirect measures. Visual Cognition, 24(2), 155-172. https://doi.org/10.1080/13506285.2016.1211209

Otsuka, S., \& Saiki, J. (2017). Neural correlates of implicit knowledge about statistical regularities. Experimental Brain Research, 235. https://doi.org/10.1007/s00221-0175083-4

Perruchet, P., \& Pacton, S. (2006). Implicit learning and statistical learning: One phenomenon, two approaches. Trends in Cognitive Sciences, 10(5), 233-238. https://doi.org/10.1016/j.tics.2006.03.006 
Reber, A. S. (1967). Implicit Learning of Artifical Grammars. Journal of Verbal Learning and Verbal Behavior, 6, 855-863.

Reber, P. J. (2013). The neural basis of implicit learning and memory: A review of neuropsychological and neuroimaging research. Neuropsychologia, 51(10), 2026-2042. https://doi.org/10.1016/j.neuropsychologia.2013.06.019

Saffran, J. R., Aslin, R. N., \& Newport, E. L. (1996). Statistical learning by 8-month-old infants. Science (New York, N.Y.), 274(5294), 1926-1928.

Saffran, J. R., \& Kirkham, N. Z. (2018). Infant Statistical Learning. Annual Review of Psychology, 69(1), 181-203. https://doi.org/10.1146/annurev-psych-122216-011805

Saffran, J. R., Newport, E. L., \& Aslin, R. N. (1996). Word Segmentation: The Role of Distributional Cues. Journal of Memory and Language, 35(4), 606-621. https://doi.org/10.1006/jmla.1996.0032

Saffran, J. R., Newport, E. L., Aslin, R. N., Tunick, R. A., \& Barrueco, S. (1997). Incidental Language Learning: Listening (and Learning) Out of the Corner of Your Ear. Psychological Science, 8(2), 101-105. https://doi.org/10.1111/j.14679280.1997.tb00690.x

Schapiro, A. C., Gregory, E., Landau, B., McCloskey, M., \& Turk-Browne, N. B. (2014). The Necessity of the Medial Temporal Lobe for Statistical Learning. Journal of Cognitive Neuroscience, 26(8), 1736-1747. https://doi.org/10.1162/jocn_a_00578

Schapiro, A. C., Kustner, L. V., \& Turk-Browne, N. B. (2012). Shaping of Object Representations in the Human Medial Temporal Lobe Based on Temporal Regularities. Current Biology, 22(17), 1622-1627. https://doi.org/10.1016/j.cub.2012.06.056

Schlichting, M. L., Guarino, K. F., Schapiro, A. C., Turk-Browne, N. B., \& Preston, A. R. (2017). Hippocampal Structure Predicts Statistical Learning and Associative Inference Abilities during Development. Journal of Cognitive Neuroscience, 29(1), 37-51. https://doi.org/10.1162/jocn_a_01028 
Siegelman, N., Bogaerts, L., Elazar, A., Arciuli, J., \& Frost, R. (2018). Linguistic entrenchment: Prior knowledge impacts statistical learning performance. Cognition, 177, 198-213. https://doi.org/10.1016/j.cognition.2018.04.011

Siegelman, N., Bogaerts, L., \& Frost, R. (2017). Measuring individual differences in statistical learning: Current pitfalls and possible solutions. Behavior Research Methods, 49(2), 418-432. https://doi.org/10.3758/s13428-016-0719-z

Siegelman, N., \& Frost, R. (2015). Statistical learning as an individual ability: Theoretical perspectives and empirical evidence. Journal of Memory and Language, 81, 105-120. https://doi.org/10.1016/j.jml.2015.02.001

Stall, S., Yarmey, L., Cutcher-Gershenfeld, J., Hanson, B., Lehnert, K., Nosek, B., ... Wyborn, L. (2019). Make scientific data FAIR. Nature, 570(7759), 27-29. https://doi.org/10.1038/d41586-019-01720-7

Stevens, D. J., Arciuli, J., \& Anderson, D. I. (2014). Concurrent Movement Impairs Incidental But Not Intentional Statistical Learning. Cognitive Science, 39(5), 1081-1098. https://doi.org/10.1111/cogs.12180

Toro, J. M., Sinnett, S., \& Soto-Faraco, S. (2005). Speech segmentation by statistical learning depends on attention. Cognition, 97(2), B25-B34. https://doi.org/10.1016/j.cognition.2005.01.006

Turk-Browne, N. B. (2012). Statistical Learning and Its Consequences. In Nebraska Symposium on Motivation. The Influence of Attention, Learning, and Motivation on Visual Search (pp. 117-146). https://doi.org/10.1007/978-1-4614-4794-8_6

Turk-Browne, N. B., Isola, P. J., Scholl, B. J., \& Treat, T. A. (2008). Multidimensional visual statistical learning. Journal of Experimental Psychology: Learning, Memory, and Cognition, 34(2), 399-407. https://doi.org/10.1037/0278-7393.34.2.399 


\section{Supplemental Methods}

22 1026.

Turk-Browne, N. B., Jungé, J. A., \& Scholl, B. J. (2005). The Automaticity of Visual Statistical Learning. Journal of Experimental Psychology: General, 134(4), 552-564. https://doi.org/10.1037/0096-3445.134.4.552

Turk-Browne, N. B., Scholl, B. J., Chun, M. M., \& Johnson, M. K. (2009). Neural evidence of statistical learning: Efficient detection of visual regularities without awareness. Journal of Cognitive Neuroscience, 21(10), 1934-1945.

Turk-Browne, N. B., Scholl, B. J., Johnson, M. K., \& Chun, M. M. (2010). Implicit Perceptual Anticipation Triggered by Statistical Learning. Journal of Neuroscience, 30(33), 1117711187. https://doi.org/10.1523/JNEUROSCI.0858-10.2010

Vakil, E., Kahan, S., Huberman, M., \& Osimani, A. (2000). Motor and non-motor sequence learning in patients with basal ganglia lesions: The case of serial reaction time (SRT). Neuropsychologia, 38(1), 1-10. https://doi.org/10.1016/S0028-3932(99)00058-5

Vallet, R. (2018). Pingouin: Statistics in Python. The Journal of Open Source Software, 3(31),

Vickery, T. J., Park, S. H., Gupta, J., \& Berryhill, M. E. (2018). Tasks determine what is learned in visual statistical learning. Psychonomic Bulletin \& Review, 25(5), 1847-1854. https://doi.org/10.3758/s13423-017-1405-6

\section{Supplemental Materials}

This section provides additional detail regarding the: Monte Carlo simulation used to generate null distributions for the direct measures, bootstrapping procedure used to generate 
1 confidence intervals for observed data, and the permutation test used to assess differences in response times by triplet position. For all simulations or resampling, the parameters were set to

3 equate the simulated/resampled data with the observed data. The number of mock participants

4 was equated to the experiment being analyzed (typically $n=120$, except Exp. 3 where $n=54$ ). The

5 number of simulations and bootstrap iterations performed was 15,000 . The number of: stimuli

6 (12), triplets (4), elements per triplet (3), and 2AFC trials (32) were the same for all experiments.

7 Code is available on the Open Science Framework (https://osf.io/vtmpb).

Monte Carlo Simulation. Determining whether the observed data was significantly

10 different than chance required estimating an unbiased null hypothesis distribution. Our approach

11 to generating such a distribution was to simulate results for mock participants across many mock

12 experiments. Once the null distribution was generated, we then determined critical thresholds

13 using our pre-registered hypotheses. We used one-tailed tests for the 2AFC and Creation

14 simulations, given that we expected learning to be above chance. In addition to the critical

15 thresholds for $\alpha=0.05$, we also calculated the critical thresholds for $\alpha=0.01 \& \alpha=0.001$. If the

16 actual mean exceeded any of three levels of significance $(p<0.05, p<0.01$, and $p<0.001)$, we

17 reported the most unlikely to have occurred by chance. Otherwise, we reported only the 18 measured value.

2AFC Simulation. An array of length 32 (i.e. number of trials) was populated with half

20 zeros and half ones to represent correct left (0) or right (1) choices. This array was used as

21 "ground truth" for all iterations. For each iteration, a mock experimental mean was calculated

22 by first calculating a mock subject mean for each fake participant, and then taking the overall 
mean of all mock participants for that iteration. The mean for a mock participant was calculated by generating an array of 32 elements (i.e. number of trials) and randomly populating it with zeros and ones. This allowed us to calculate the "number 2AFC correct" for each mock participant. After all 15,000 iterations completed, we sorted the mock experimental means into ascending order to reveal the null distribution, calculating the overall chance experimental mean and a critical threshold for "chance" performance, using an alpha of 0.05 (i.e. taking the value at 0.95 as the critical threshold).

Creation Simulation. This simulation was used to calculate chance performance for both triplets created and foils created. An array of length 12 (i.e. number of stimuli) was populated with unique values from 0-11 (inclusive) to represent the stimulus identities. This array was used as "ground truth" for all iterations. Triplets were defined by splitting these 12 values into sets of three (i.e. " 0 ", "1", " 2 " is triplet 1 ). For each iteration, a mock experimental mean was calculated. In each iteration, we looped through each fake participant and calculated the mock experimental mean by generating an array of 12 elements (i.e. number of stimuli) and randomly populated it with each value from 0-11. We then split the random 12 values into sets of three, allowing us to calculate the "number correct" for each mock participant. After all 15,000 iterations completed, we sorted the values in ascending order to create a null distribution. From this distribution, we calculated the overall chance experimental mean and a critical threshold for "chance" performance, using an alpha of 0.05 (i.e. taking the value at 0.95 as the critical threshold).

Bootstrap Procedure. 95\% confidence intervals were calculated using a bootstrap procedure. We ran multiple "mock experiments". In each mock experiment, we sampled randomly (with replacement) from the actual experimental data (e.g. from the list of 2AFC 
percent-correct scores across participants) to generate a surrogate pool of 2AFC values, and computed the mean 2AFC performance for that mock experiment. Finally, we sorted the means from all the mock experiments in ascending order, employing the 2.5 and 97.5 percentiles as the boundaries of the $95 \%$ confidence interval.

Permutation Testing. For comparing whether there was a significant difference between response times in the first and later triplet positions, we used the following permutation test procedure. Response times (RTs) were combined into one of two groups: first and second triplet positions or first and third triplet positions. RTs were then randomly selected (with replacement) into two mock choice arrays, with each array equal to the number of TD trials (72). The difference between the means of these arrays became the estimate for either RTF1-2 (first - second triplet position) or RTF1-3 (first - third triplet position) for a mock participant. Note that the RT facilitation measurements are not simply the differences between mean RTs across all mock participants, but rather the mean of within-subject facilitation, thus eliminating variance in the mean RT across participants, and producing a more powerful test of RT facilitation. The RTF means of all mock participants were then sorted into ascending order. Next, we determined the chance location for three levels of significance $(p<0.05, p<0.01$, and $p<0.001)$ using a one-tailed test. The level of significance (e.g. $p=0.05$ ) was multiplied by the number of iterations to determine the index of the critical comparison value. If the actual mean exceeded any of these three threshold levels, we reported the most unlikely to have occurred by chance. Otherwise, we reported only the measured value.

Models of multiple linear regression. We created two linear models to assess the impact of independent variables on response times in the target detection task. The simple model 
1

contained stream position as the only predictor of response time, while the more complex model contained both and stream position and triplet position as linear predictors of response time. We compared the simple model and complex model to one another.

4

Exclusion Methodology. Exclusion criteria are grouped into three categories: technical issues, insufficient attention, and issues with instructions. In our experiments, participants were excluded if: (1) the data was unreadable through the standard analysis pipeline or otherwise recorded incorrectly (Participants Excluded - Data Corrupted or Recorded Incorrectly) or (2) if they participated in the experiment for a duration exceeding the maximum allowed duration (Participants Excluded - Experimental Duration Exceeded). We use multiple attentional checks throughout our experiments, including checks for: insufficient elements detected in the cover task, insufficient targets detected during TD task, excessive switching tabs outside of the experiment, or insufficient number of choices made during the 2AFC task (Participants Excluded - Insufficient Jiggles Caught , Participants Excluded - Insufficient Targets Caught, Participants Excluded - Excessive "Focus Off" Events, and Participants Excluded - Insufficient 2AFC Choices). The final category of exclusions includes any participants who had difficulty understanding or executing the instructions. This set of conditions include participants who: did not attend to all images, attempted to participate more than once, produced an excessive number of keypresses, indicated they did not understand the instructions, or otherwise made statements indicating they did not understand the instructions (Participants Excluded - Could Not See All Images, Participants Excluded - Previously Participated in Similar Experiment, Participants Excluded -

21 Excessive Keypresses, Participants Excluded - Did Not Understand Instructions, Participants 2 Excluded - Disregarded Instructions) (Supp. Table 1). 
Counterbalancing procedure for stimulus sets. Counterbalancing was achieved by creating "mini-groups" of three stimulus sets, where the item positions were circularly shifted

3 within a triplet across stimulus sets. Thus, subject $X$ might be assigned the first stimulus set in a

4 mini-group, and would see a triplet composed of the stimuli: $\left(A_{1}, A_{2}, A_{3}\right)$. Subject $Y$ might be

5 assigned the second stimulus set in the mini-group, and would see a triplet composed of the

6 stimuli: $\left(A_{2}, A_{3}, A_{1}\right)$. Finally, subject $Z$, would see a triplet composed of: $\left(A_{3}, A_{1}, A_{2}\right)$. In this way,

7 each stimulus image, $A_{1}$ or $A_{2}$ or $A_{3}$, appears equally in the first, second and third position of a

8 triplet. This circular shifting procedure ensures triplet position is uncorrelated with the properties

9 of individual stimuli.

10

11 Supplemental Figures \& Tables

12

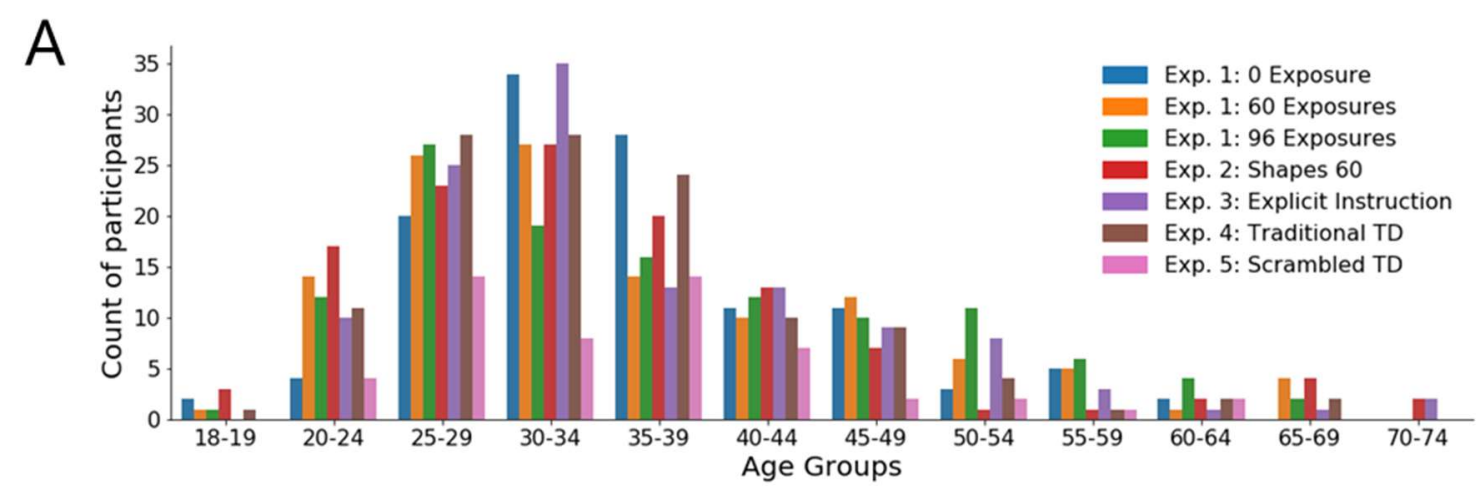

B

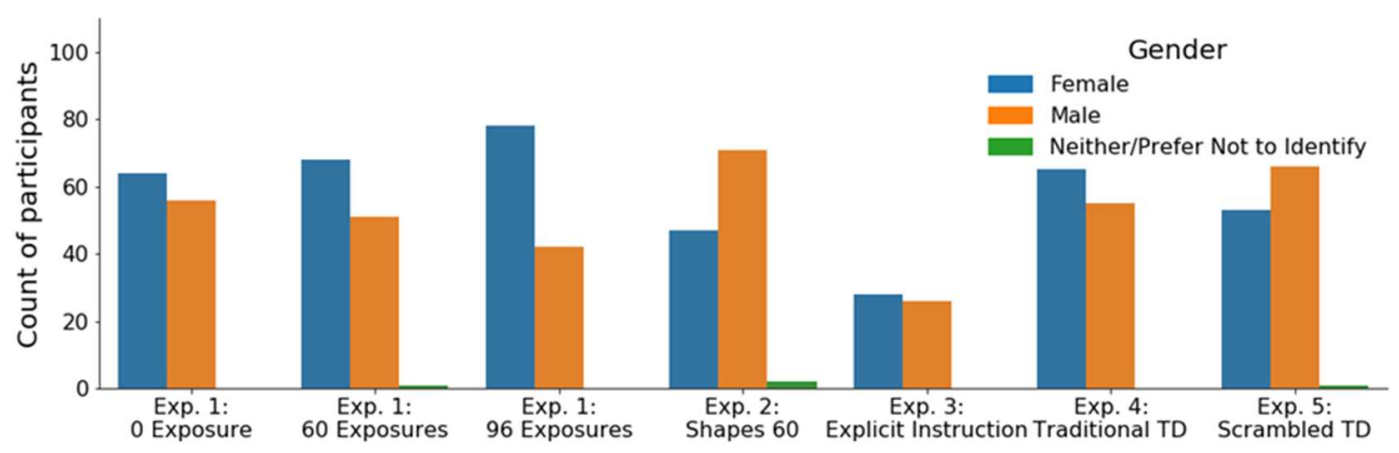


1 Supp. Fig. 1. Distribution of participants by age group and gender. (A) Histogram of participants by age group. (B)

2 Histogram of participants by gender.

3

4

5 Supplemental Table 1

6 Experimental Parameters

\begin{tabular}{|c|c|c|c|c|c|c|c|}
\hline $\begin{array}{l}\text { Experiment } \\
\text { Name }\end{array}$ & $\begin{array}{l}\text { Fractals: } 0 \\
\text { Exposure }\end{array}$ & $\begin{array}{l}\text { Fractals: } 60 \\
\text { Exposures }\end{array}$ & $\begin{array}{l}\text { Fractals: } 96 \\
\text { Exposures }\end{array}$ & Shapes & $\begin{array}{l}\text { Explicit } \\
\text { Instruction }\end{array}$ & $\begin{array}{l}\text { Traditional } \\
\text { TD }\end{array}$ & $\begin{array}{l}\text { Scrambled } \\
\text { TD }\end{array}$ \\
\hline $\begin{array}{l}\text { Experiment } \\
\text { Number }\end{array}$ & 1 & 1 & 1 & 2 & 3 & 4 & 5 \\
\hline \# Analyzed & 120 & 120 & 120 & 120 & 54 & 120 & 120 \\
\hline $\begin{array}{l}\text { \# Excluded - } \\
\text { Data Corrupted } \\
\text { or Recorded } \\
\text { Incorrectly }\end{array}$ & 1 & 0 & 0 & 1 & 1 & 1 & 1 \\
\hline $\begin{array}{l}\text { \# Excluded - } \\
\text { Insufficient } \\
\text { Jiggles Caught }\end{array}$ & 0 & 10 & 13 & 2 & 0 & 9 & 9 \\
\hline $\begin{array}{l}\text { \# Excluded - } \\
\text { Insufficient } \\
\text { Targets Caught }\end{array}$ & 3 & 4 & 3 & 2 & 5 & 11 & 9 \\
\hline $\begin{array}{l}\text { \# Excluded - } \\
\text { Excessive "Focus } \\
\text { Off" Events }\end{array}$ & 5 & 7 & 10 & 2 & 16 & 10 & 7 \\
\hline $\begin{array}{l}\text { \# Excluded - } \\
\text { Could Not See } \\
\text { All Images }\end{array}$ & 0 & 3 & 4 & 1 & 0 & 8 & 6 \\
\hline $\begin{array}{l}\text { \# Excluded - } \\
\text { Previously } \\
\text { Participated in } \\
\text { Similar } \\
\text { Experiment }\end{array}$ & 3 & 3 & 7 & 0 & 1 & 11 & 9 \\
\hline $\begin{array}{l}\text { \# Excluded - } \\
\text { Excessive } \\
\text { Keypresses }\end{array}$ & 5 & 4 & 6 & 3 & 2 & 20 & 9 \\
\hline $\begin{array}{l}\text { \# Excluded - } \\
\text { Insufficient 2AFC } \\
\text { Choices }\end{array}$ & 15 & 4 & 2 & 1 & 18 & 5 & 11 \\
\hline $\begin{array}{l}\text { \# Excluded - Did } \\
\text { Not Understand } \\
\text { Instructions }\end{array}$ & 9 & 9 & 9 & 2 & 2 & 14 & 13 \\
\hline $\begin{array}{l}\text { \# Excluded - } \\
\text { Disregarded } \\
\text { Instructions }\end{array}$ & 0 & 7 & 8 & 3 & 0 & 11 & 4 \\
\hline $\begin{array}{l}\text { \# Excluded - } \\
\text { Experimental } \\
\text { Duration } \\
\text { Exceeded }\end{array}$ & 0 & 0 & 3 & 1 & 0 & 2 & 0 \\
\hline
\end{tabular}




\begin{tabular}{|c|c|c|c|c|c|c|c|}
\hline $\begin{array}{l}\text { \# Not Analyzed } \\
\text { (i.e. "Held Out") }\end{array}$ & 41 & 13 & 31 & 38 & 8 & 46 & 12 \\
\hline Compensation & $\$ 2.75$ & $\$ 4.50$ & $\$ 5.38$ & $\$ 4.40$ & $\$ 4.40$ & $\$ 4.40$ & $\$ 4.40$ \\
\hline $\begin{array}{l}\text { Initial Exposure } \\
\text { Stimulus } \\
\text { Duration (ms) }\end{array}$ & $\mathrm{n} / \mathrm{a}$ & 800 & 800 & 800 & 800 & 800 & 800 \\
\hline $\begin{array}{l}\text { Initial Exposure } \\
\text { Inter-stimulus } \\
\text { Interval (ms) }\end{array}$ & $\mathrm{n} / \mathrm{a}$ & 800 & 800 & 200 & 200 & 200 & 200 \\
\hline $\begin{array}{l}\text { TD Stimulus } \\
\text { Duration (ms) }\end{array}$ & 200 & 200 & 200 & 200 & 200 & 200 & 200 \\
\hline $\begin{array}{l}\text { TD Inter- } \\
\text { stimulus } \\
\text { Duration (ms) }\end{array}$ & 40 & 40 & 40 & 40 & 40 & 40 & 40 \\
\hline $\begin{array}{l}\text { \# Elements per } \\
\text { TD trial }\end{array}$ & $7-11$ & $7-11$ & $7-11$ & $7-11$ & $7-11$ & 12 & 12 \\
\hline Cover Task & $\mathrm{n} / \mathrm{a}$ & Jiggle & Jiggle & Jiggle & $\begin{array}{l}\text { Explicit } \\
\text { Instruction }\end{array}$ & Jiggle & Jiggle \\
\hline Stimulus Type & Fractals & Fractals & Fractals & Shapes & Fractals & Fractals & Fractals \\
\hline $\begin{array}{l}\text { Mean } \\
\text { percentage of } \\
\text { jiggles caught }\end{array}$ & $\mathrm{n} / \mathrm{a}$ & $90 \%$ & $90 \%$ & $91 \%$ & $\mathrm{n} / \mathrm{a}$ & $92 \%$ & $92 \%$ \\
\hline $\begin{array}{l}\text { Mean jiggle } \\
\text { response time } \\
\text { (ms) }\end{array}$ & $\mathrm{n} / \mathrm{a}$ & 446 & 458 & 447 & $\mathrm{n} / \mathrm{a}$ & 445 & 442 \\
\hline $\begin{array}{l}\text { Mean } \\
\text { percentage of } \\
\text { targets caught }\end{array}$ & $90 \%$ & $90 \%$ & $89 \%$ & $92 \%$ & $86 \%$ & $89 \%$ & $91 \%$ \\
\hline $\begin{array}{l}\text { Mean target } \\
\text { response time } \\
\text { (ms) }\end{array}$ & 424 & 410 & 414 & 394 & 417 & 433 & 439 \\
\hline $\begin{array}{l}\text { TD Response } \\
\text { Time Split Half } \\
\text { Reliability }(r)\end{array}$ & $\begin{array}{l}0.73, \\
p<0.001\end{array}$ & $\begin{array}{l}0.80 \\
p<0.001\end{array}$ & $\begin{array}{l}0.73, \\
p<0.001\end{array}$ & $\begin{array}{l}0.74, \\
p<0.001\end{array}$ & $\begin{array}{l}0.69 \\
p<0.001\end{array}$ & $\begin{array}{l}0.82, \\
p<0.001\end{array}$ & $\begin{array}{l}0.78, \\
p<0.001\end{array}$ \\
\hline $\begin{array}{l}\text { 2AFC Split Half } \\
\text { Reliability (r) }\end{array}$ & $\begin{array}{l}0.37 \\
p<0.001\end{array}$ & $\begin{array}{l}0.29, \\
p=0.0015\end{array}$ & $\begin{array}{l}0.24, \\
p=0.0080\end{array}$ & $\begin{array}{l}0.44 \\
p<0.001\end{array}$ & $\begin{array}{l}0.74 \\
p<0.001\end{array}$ & $\begin{array}{l}0.37 \\
p<0.001\end{array}$ & $\begin{array}{l}0.22, \\
p=0.015\end{array}$ \\
\hline $\begin{array}{l}\text { TD Stream } \\
\text { Position RT slope } \\
\text { (ms/item) }\end{array}$ & -18.3 & -18.1 & -16.8 & -20.2 & -16.9 & -15.4 & -15.3 \\
\hline
\end{tabular}




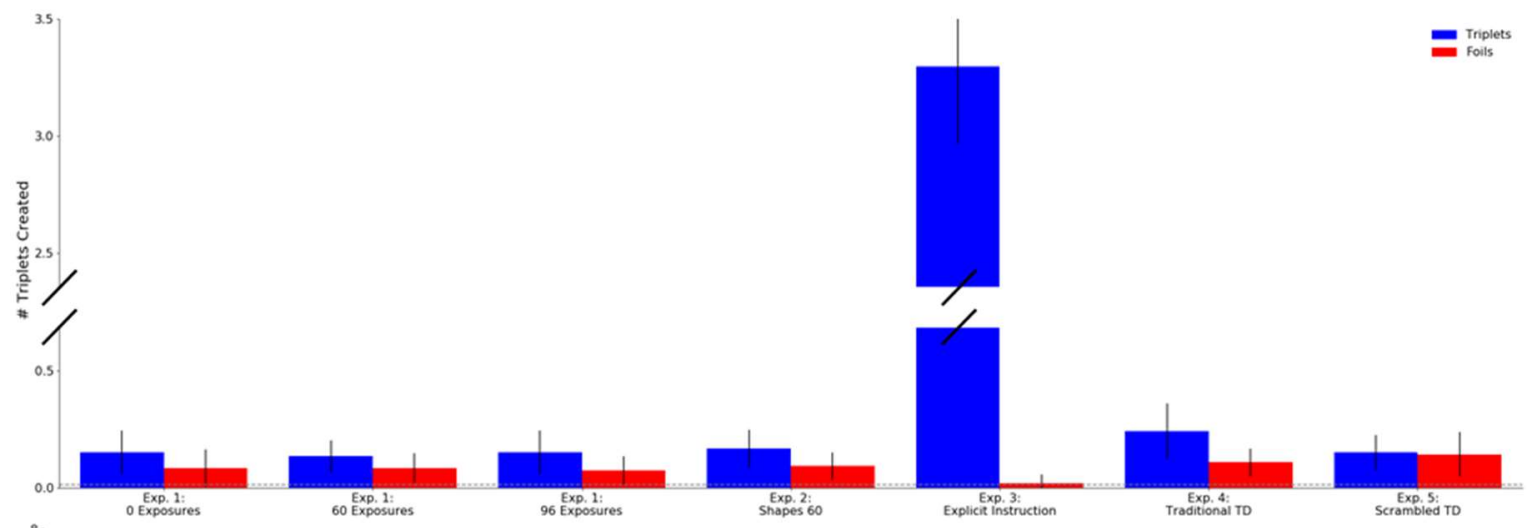

B
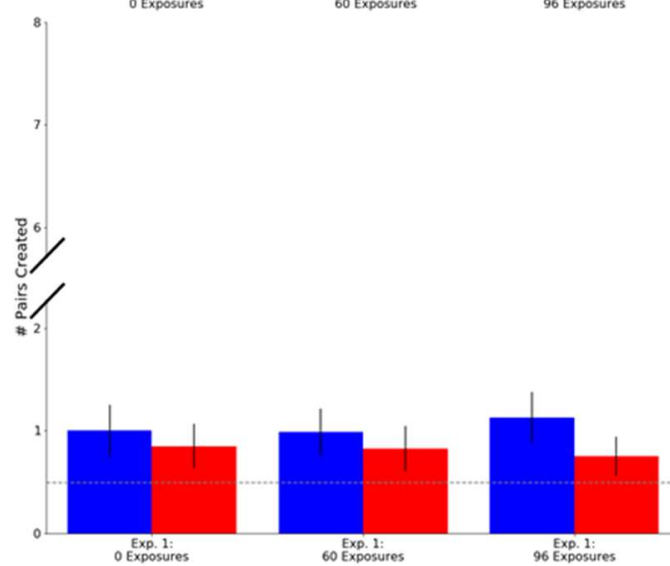

96 Exposives

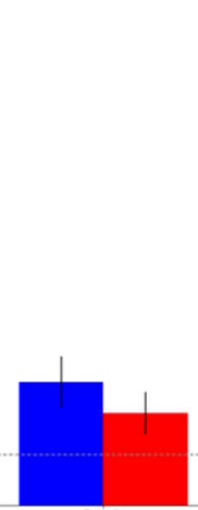

Shop:260

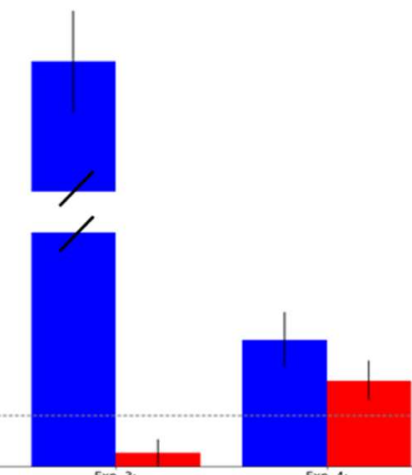

Explexichingsivection

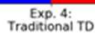

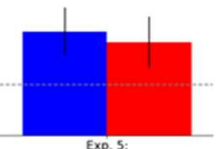

Supp. Fig. 2. Performance in Creation task. (A) Number of triplets created that were "real" (i.e. triplets presented during exposure and/or TD task) and foil triplets from the 2AFC. (B) Number of pairs created that were "real" (i.e. a two-element sequence that was part of a triplet presented during exposure and/or TD) and foil pairs from the 2AFC. Dashed lines indicate chance mean, estimated using Monte Carlo simulation.
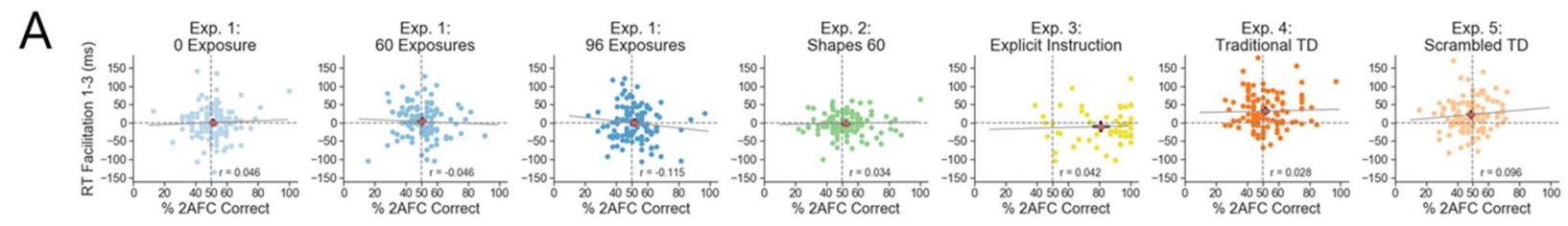

B
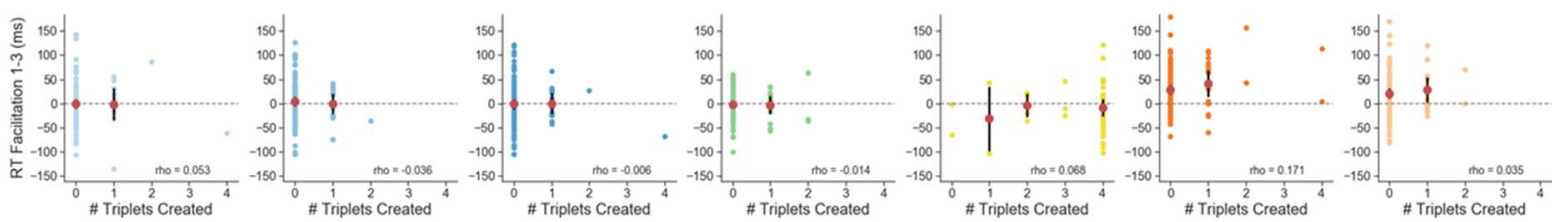

Supp. Fig. 3. Relationships between indirect and direct measures of Visual Statistical Learning. (A) Relationship between response time facilitation between first and third triplet positions and percentage of 2AFC trials correctly chosen. (B) Relationship between response time facilitation between first and third triplet positions and number of triplets successfully created. Means are shown in red with black line segments indicating $95 \% \mathrm{Cls}$ (where $>4$ data points exist). Pearson correlation shown in (A), Spearman correlation shown in (B). 

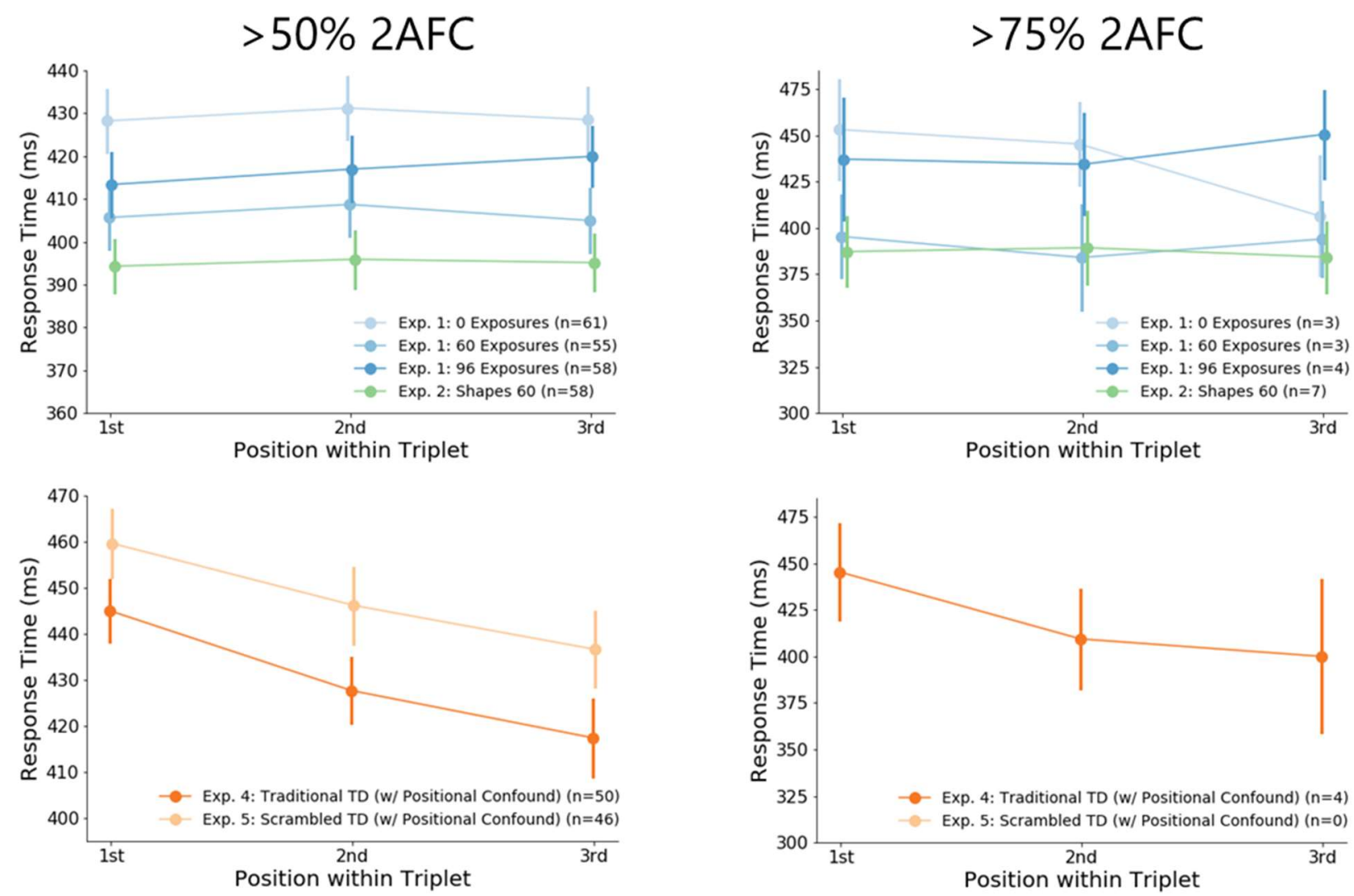

Supp. Fig. 4. Response times by triplet position for "learners". (left column) Participants who were above chance

3 (50\%) in 2AFC performance. (right column) Participants who were above a statistically significant bootstrap chance 4 level (75\%) in 2AFC performance. Note there were no participants in Experiment 5 with $>75 \% 2 A F C$ performance.

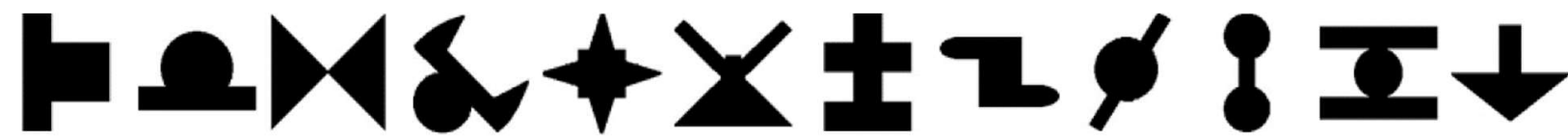

7 Supp. Fig. 5. Stimuli used in Experiment 2 (Shapes). 

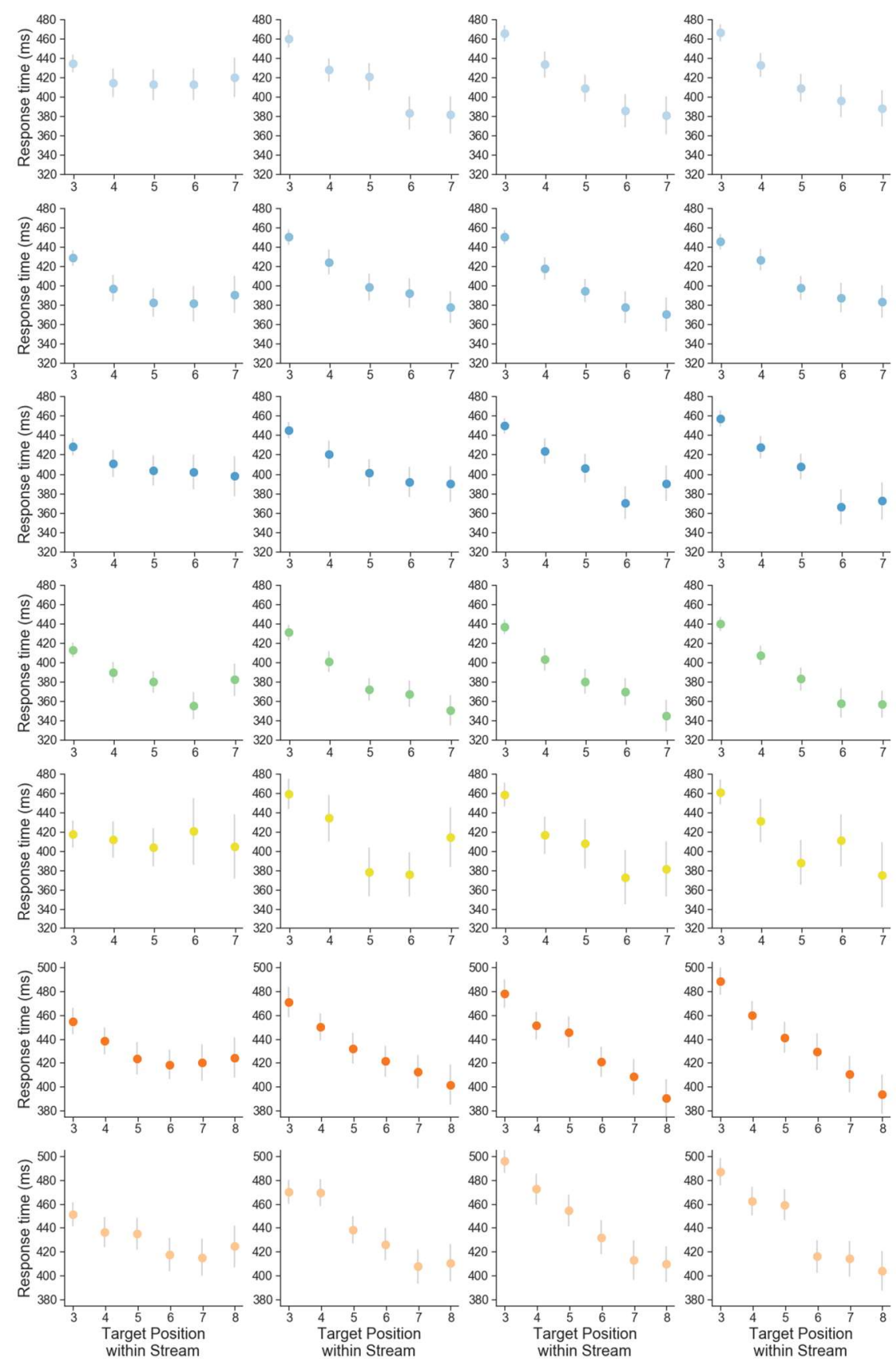

Supp. Fig. 6. Response times by stream position binned by TD trial quartile (each column contains 18 trials). (top 3 row) Experiment 1: 0 Exposure. (second row) Experiment 1: 60 Exposures. (third row) Experiment 1: 96 Exposures.

4 (fourth row) Experiment 2: Shapes. (fifth row) Experiment 3: Explicit Instructions. (sixth row) Experiment 4:

5 Traditional TD. (bottom row) Experiment 5: Scrambled TD.

6

7 Supplemental Table 2

$8 \quad$ Average decrease ( $m s$ ) between stream positions (by quartile)

\begin{tabular}{llccc}
\hline & Trials 1-18 & Trials 19-36 & Trials 37-54 & Trials 55-72 \\
\hline Exp. 1: 0 Exposure & 3.6 & 19.7 & 21.1 & 19.6
\end{tabular}




$\begin{array}{lllll}\text { Exp. 1: 60 Exposures } & 9.4 & 18.2 & 20.0 & 15.4 \\ \text { Exp. 1: 96 Exposures } & 7.5 & 13.9 & 14.9 & 21.3 \\ \text { Exp. 2: Shapes 60 } & 7.8 & 20.1 & 22.9 & 20.7 \\ \text { Exp. 3: Explicit Instruction } & 3.2 & 11.1 & 19.1 & 21.4 \\ \text { Exp. 4: Traditional TD } & 6.1 & 13.9 & 17.6 & 18.9 \\ \text { Exp. 5: Scrambled TD } & 5.4 & 11.9 & 17.3 & 16.6\end{array}$
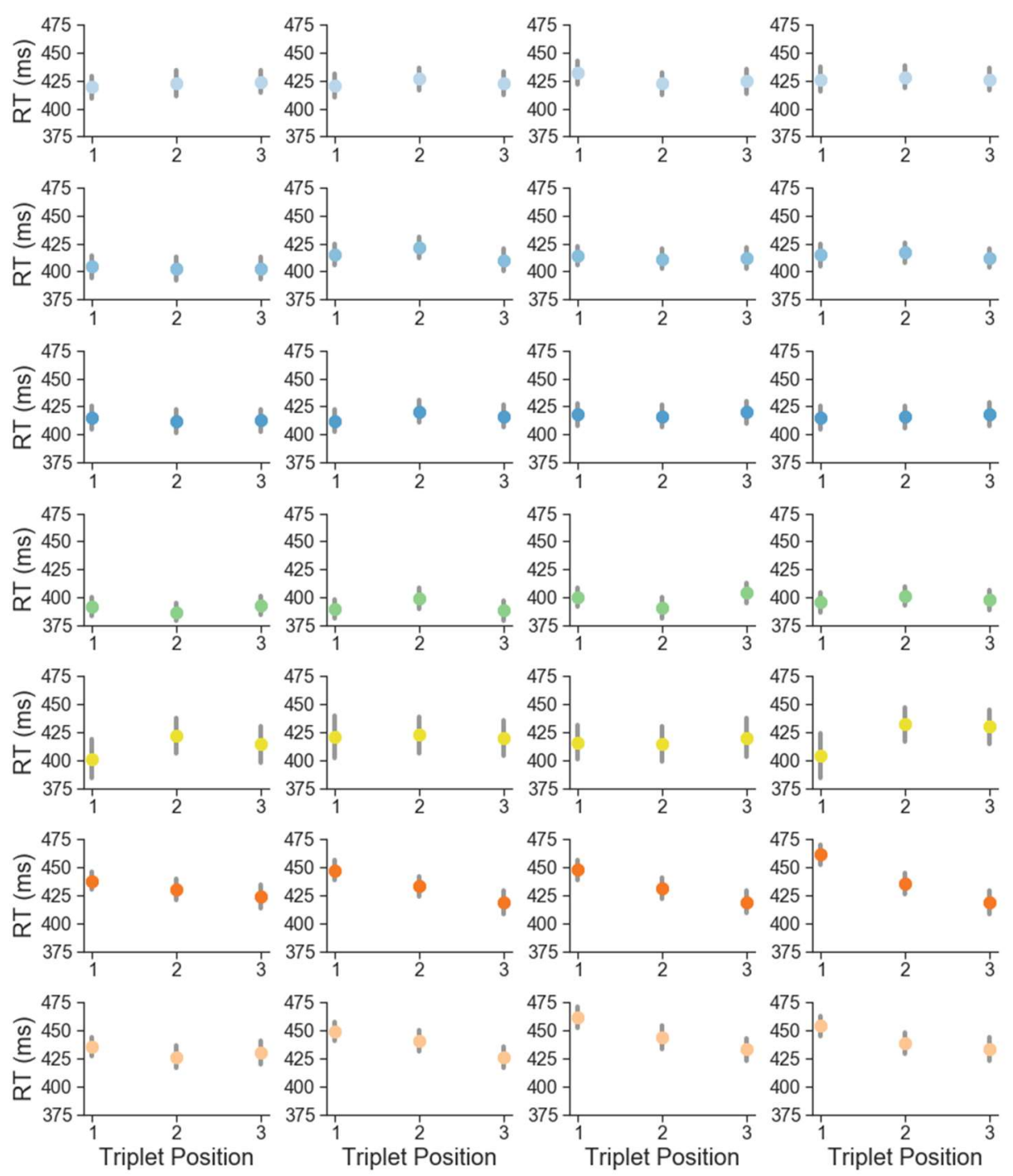

4 Supp. Fig. 7. Response times by triplet position binned by quartile of TD trials (each column contains 18 trials).

5 (top row) Experiment 1: 0 Exposure. (second row) Experiment 1:60 Exposures. (third row) Experiment 1: 96

6 Exposures. (fourth row) Experiment 2: Shapes. (fifth row) Experiment 3: Explicit Instructions. (sixth row)

7 Experiment 4: Traditional TD (bottom row) Experiment 5: Scrambled TD. 
1

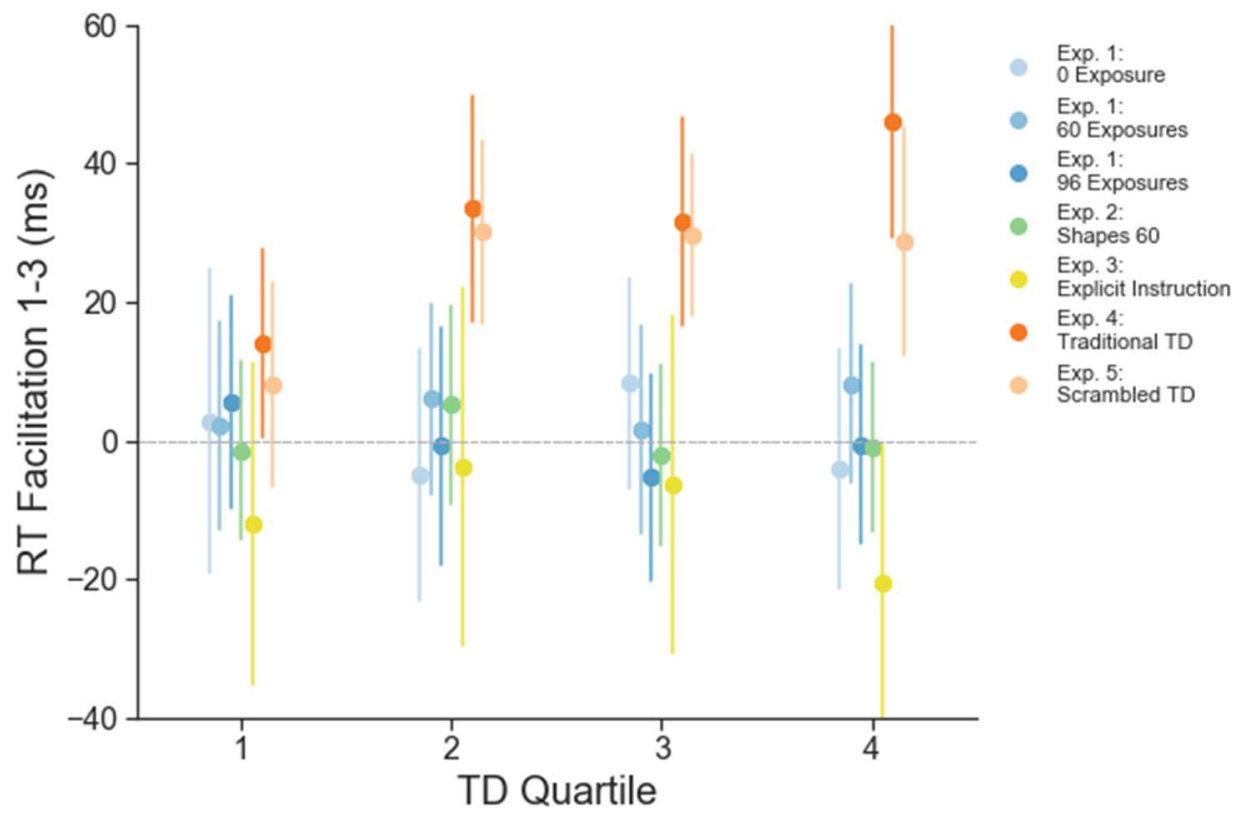

2

3 Supp. Fig. 8. Response time facilitation between first and third triplet positions binned by TD trial quartile. Each 4 quartile contains 18 trials.

5

6

7

8 


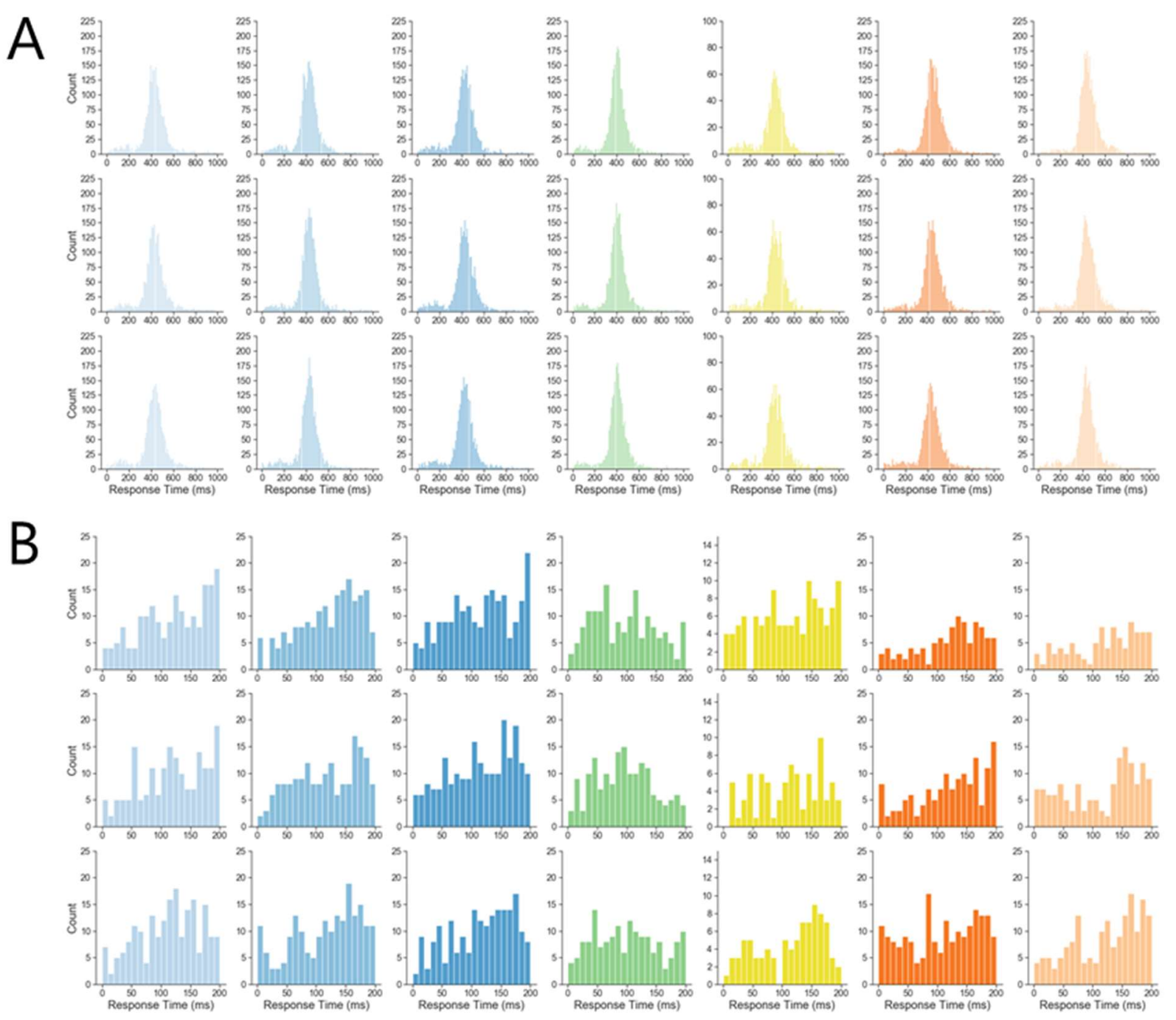

Supp. Fig. 9. Target Detection task response times by experiment and triplet position. (A\&B first rows) Response times for triplet position 1. (A\&B middle rows) Response times for triplet position 2. (A\&B bottom rows) Response times for triplet position 3. (A) Overall distribution of response times. (B) Sub-200ms response times only.

Supplemental Notes

No RT facilitation differences between Experiments 4 \& 5. If the stream position effect

9 accounts for RT facilitation, then why was there a numerically larger response time facilitation in Experiment 4 (Traditional TD) than in Experiment 5 (Scrambled TD) (Fig. 6A)? In fact, the RT

11 facilitation (position 1 minus position 3 RT) was not significantly different across the two 12 experiments $(W=3073, M P R B C=-0.13$; Supp. Figs. 3-5). However, if the numerical difference was 
not just a statistical fluctuation, then one possible explanation for the difference would some kind of learning during the TD task in Experiment 4. Given that the exposure phases were identical between Experiments 4 \& 5, it seemed unlikely that such learning could have arisen from regularities seen during the exposure phase.

Participants with poor performance in 2AFC task, despite explicit knowledge. Although learning in Experiment 3 was strong (with 50\% of participants achieving $>90 \% 2 \mathrm{AFC}$ ), one-third of participants did not achieve 2AFC accuracy $>=75 \%$. Previous work has shown that around onethird of participants demonstrate chance performance in statistical learning tasks (Siegelman \& Frost, 2015), so it is possible that a subset of individuals are unable to express learning under standard 2AFC testing.

Learning During Test. Consistent with the notion that some form of learning occurred during the TD task, the stream position effect increased as participants saw more trials (Supp. Fig. 6; Supp. Table 2). Because of stream position speeding effect in the TD task, triplet position effects increased in Experiments 4 \& 5, but not in Experiments 1-3 (Supp. Fig. 7). As a result, response time facilitation (RTF1-3) effects increased from the early trials to the later trials in Experiments 4 \& 5 (see "No RT facilitation differences between Experiments 4 \& 5" in Supp.), but not Experiments 1-3, (Supp. Fig. 8). Participants could learn the structure of the TD task even in the absence of any sequential regularities in the visual stream (Fig. 6D). The Scrambled TD in Experiment 5, which contained no statistical regularities, showed no statistically significant change in RT facilitation across quartiles $(H(3)=5.53$; Supp. Fig. 9), but if this approximately $22 \mathrm{~ms}$ change from the first to later quartiles is meaningful, it would suggest that participants response times are speeded after learning about task features other than triplet regularities: for example, 
1 by learning that targets can only occur in the $4^{\text {th }}$ through $8^{\text {th }}$ items in the steam. These follow-up

2 analyses suggest that RT facilitation in the traditional TD task (Exp. 4, Fig. 6A) could arise from

3 actual triplet learning, but here arises primarily from a mixture of two sources: (i) the stream

4 position effect and (ii) learning of task structure during test, rather than during initial exposure.

5 More explicit forms of knowledge may also arise from engagement with indirect tests like the

6 TD task, which contain triplet regularities. In the "0 Exposure" condition of Experiment 1, there

7 was no initial exposure phase, and yet 2AFC and Creation performance were above chance

8 performance $(2 \mathrm{AFC}$ mean $=51.7,95 \% \mathrm{Cl}=[51.5,51.9], \mathrm{p}=0.018$; Creation mean $=0.150,95 \%$

$9 \mathrm{Cl}=[0.142,0.158], \mathrm{p}<0.001)$. Therefore, learning could only have occurred during the TD task. In

10 contrast, no learning was observed during test for the direct measures, with performance in the

11 2AFC task remaining consistent from the first to second half of trials ("2AFC Split Half

12 Reliability", Supp. Table 1).

13 This "learning during test" could be a form of procedural learning, perhaps analogous to

14 serial reaction time (SRT) learning, which is known to be basal-ganglia dependent (Vakil, Kahan,

15 Huberman, \& Osimani, 2000) and is thought to be qualitatively distinct from standard statistical

16 learning (Siegelman \& Frost, 2015). Future work should investigate how engagement with

17 regularities contributes to the extraction of regularities.

Stream Length in Target Detection Tasks. In designs where streams are lengthened to 20

or more items, response times do not monotonically decrease across all stream positions

20 (Batterink, 2017; c.f. Figs. 4A; 6D). In such designs, our preliminary analyses (data not shown)

21 suggest that a variant of the stream position effect is still present: response times decrease

22 monotonically with the number of images after the last target presentation. Importantly, 
1 however, in these designs in which targets repeat within a stream, the stream positions and

2 triplet positions are not perfectly confounded: thus, for auditory tasks with long test-streams

3 (Batterink, 2017) triplet position effects cannot be explained by a confounding effect of stream 4 position. 